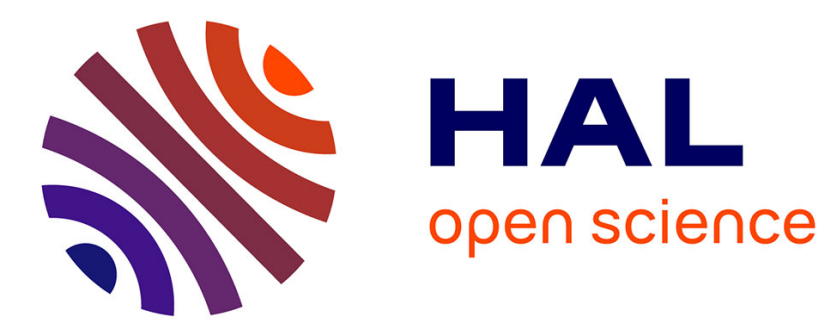

\title{
Fouilles à la plate-forme de Forum Julii (Fréjus, Var) en 1960 et 1961
}

\author{
Paul-Albert Février
}

\section{To cite this version:}

Paul-Albert Février. Fouilles à la plate-forme de Forum Julii (Fréjus, Var) en 1960 et 1961. Gallia - Fouilles et monuments archéologiques en France métropolitaine, 1962, 20 (1), pp.177-203. 10.3406/galia.1962.2353 . hal-01931207

\section{HAL Id: hal-01931207 https://hal.science/hal-01931207}

Submitted on 25 Feb 2020

HAL is a multi-disciplinary open access archive for the deposit and dissemination of scientific research documents, whether they are published or not. The documents may come from teaching and research institutions in France or abroad, or from public or private research centers.
L'archive ouverte pluridisciplinaire HAL, est destinée au dépôt et à la diffusion de documents scientifiques de niveau recherche, publiés ou non, émanant des établissements d'enseignement et de recherche français ou étrangers, des laboratoires publics ou privés.

\section{(ㅇ)(1) $\$$}

Distributed under a Creative Commons Attribution - NonCommercial - NoDerivatives| 4.0 


\section{GOUILIEA I IA PIATE-FORME IOE FORUM JULII (Fréjus, Var) on 1960 ol 19611 \\ par Paul-Alluert Fívilik}

Lans la partir orientale de la cilé a l'angle sudtest de l'enceinte, une construction domine le port romain et la plaine d'une dizaine de metres : cest l'édifiece appelé traditionnellement la Plate-Forme. Xous lui conserverons en nom faule de pouvoir identifier eneore rorrectement le monument. Iess murs qui délimitent la butte et soutiennent les lerres ont depuis longtemps attiré l'attention ${ }^{2}$. I)es 1803, Villeneuve-Bargemont entreprit à l'instigation du préfet Fauchet le déblair ment de la citerne qui est au centre et il en fournit une description dans son rapport ${ }^{3}$. A la fin du $x_{1} x^{e}$ siècle, Aubenas fit une fouille en un point mal précisé, sans doute vers l'angle sud-est'. C'est au docteur Ionnadieu que revient le mérite d'avoir mené une série de campagnes de 1930 à 1939, qui firent connaitre les grandes lignes du plan. Ces fouilles n'ont pas été suivies d'une publication romplete; scules quelques notes subsistent.5. Le terrain est resté ahandonné, ce qui a nui à la conservation du monument. Cne fois le terrain acheté par l'État, il a été possible de reprendre de façon svistémalique ces travaux et le dégagement de la totalité du monument a été commencé en octolire 1960.

\section{Les murs de soulènement.}

I à où les Romains établirent le monument de la Plate-Forme existait à l'origine une butte naturelle. Nulle part encore, les sondages n'ont fait apparaitre le rocher. Seules des couches vierges d'argile. de sable, ou de sable et d'argile mèlés ont été reconnues. Les tranchées creusées réemment dans un terrain voisin à l'extérieur de la ville vers l'Est font apparaître le même sous-sol. Les sondages pratiqués jusqu'à présent et l'étude des murs de soutenement montrent que cette butte offrait vers l'Ouest une pente assez douce. A l'Est at au sud. le relief était plus sensible. Pour régulariser la butte et établir le terreplein. les Romains utilisèrent l'enceinte de la cité et hàtirent des murs de soutènement.

(1) V. sur les fouilles précédentes de Fréjus, Gallia, XIV, 1956, p. 35-i33; XVIII, 1960, p. 313.

(2) Forma orbis romani, Carte archéologique de la Gaule romaine, fasc.II, Paris, 1932, no 4, p. 2-3.

(3) Chr. de Vildenflue-Bargenoxt, Rapport présente..., dans Mém. de la soc. agr. des sciences el arls d'Agen, t. II, 1812

(4) J.-A. Arbrisas, Histoire de Fréjus, Fréjus, 1881 , p. xi-xiv.

(5) J. Doxxante dans Bull. arch. du com., 1930-1931, p.506-507; A. Doxisadfu, dans Congress arch de France,

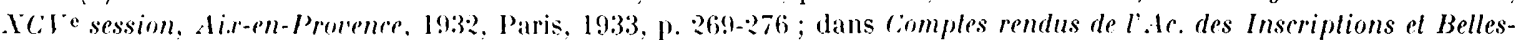
Lellres, 1932, p. 115-118; dans Bull. arch. du com., 1933-1910, p. 177-188; Bull. de la soc. d'ét. de Draguignan, t. XI., 1931-1935, p. 106-108. Ajouter un rapport dact ylographie à la Bibl. des .Mon. hist., ms. 3, 1937. 


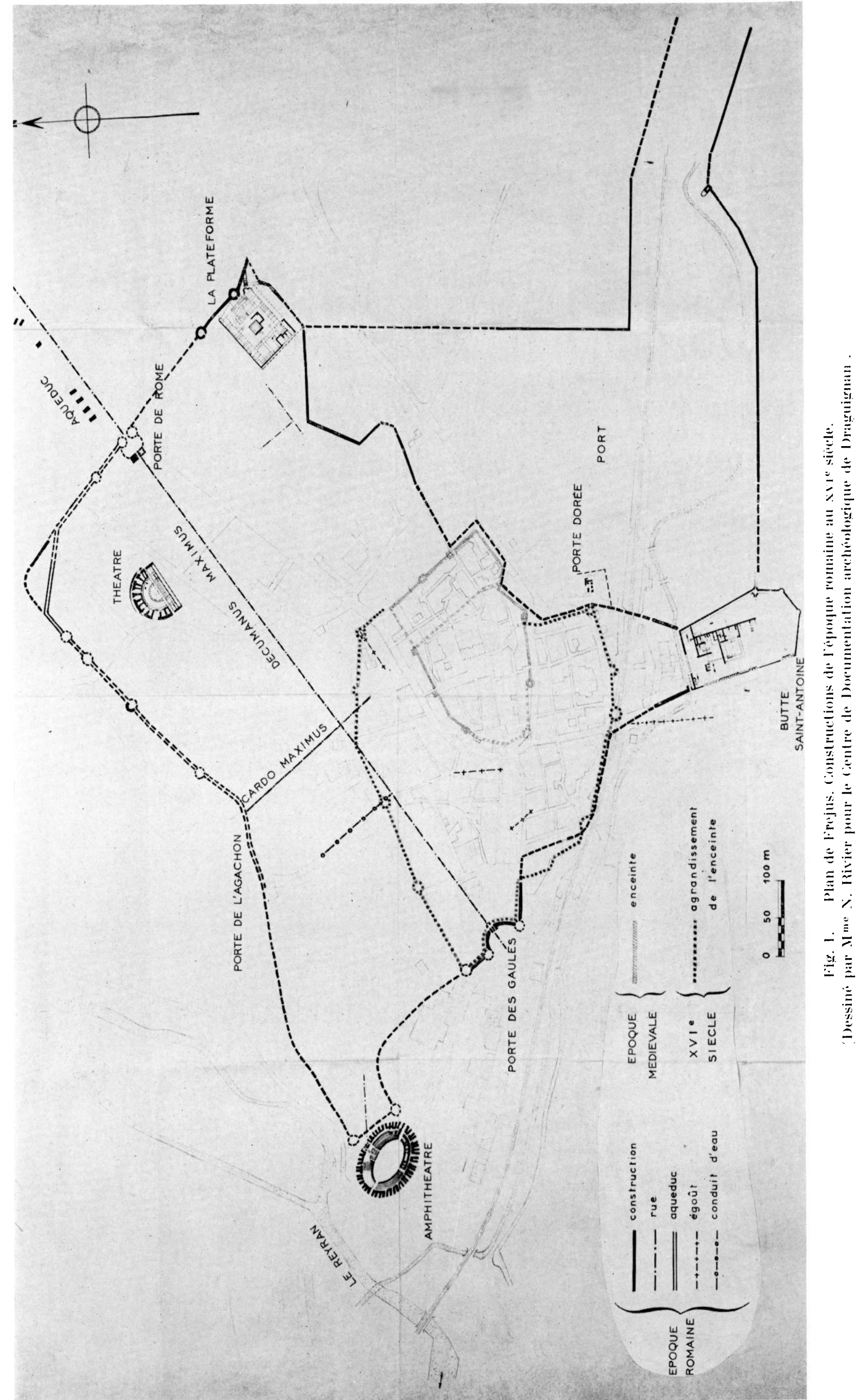




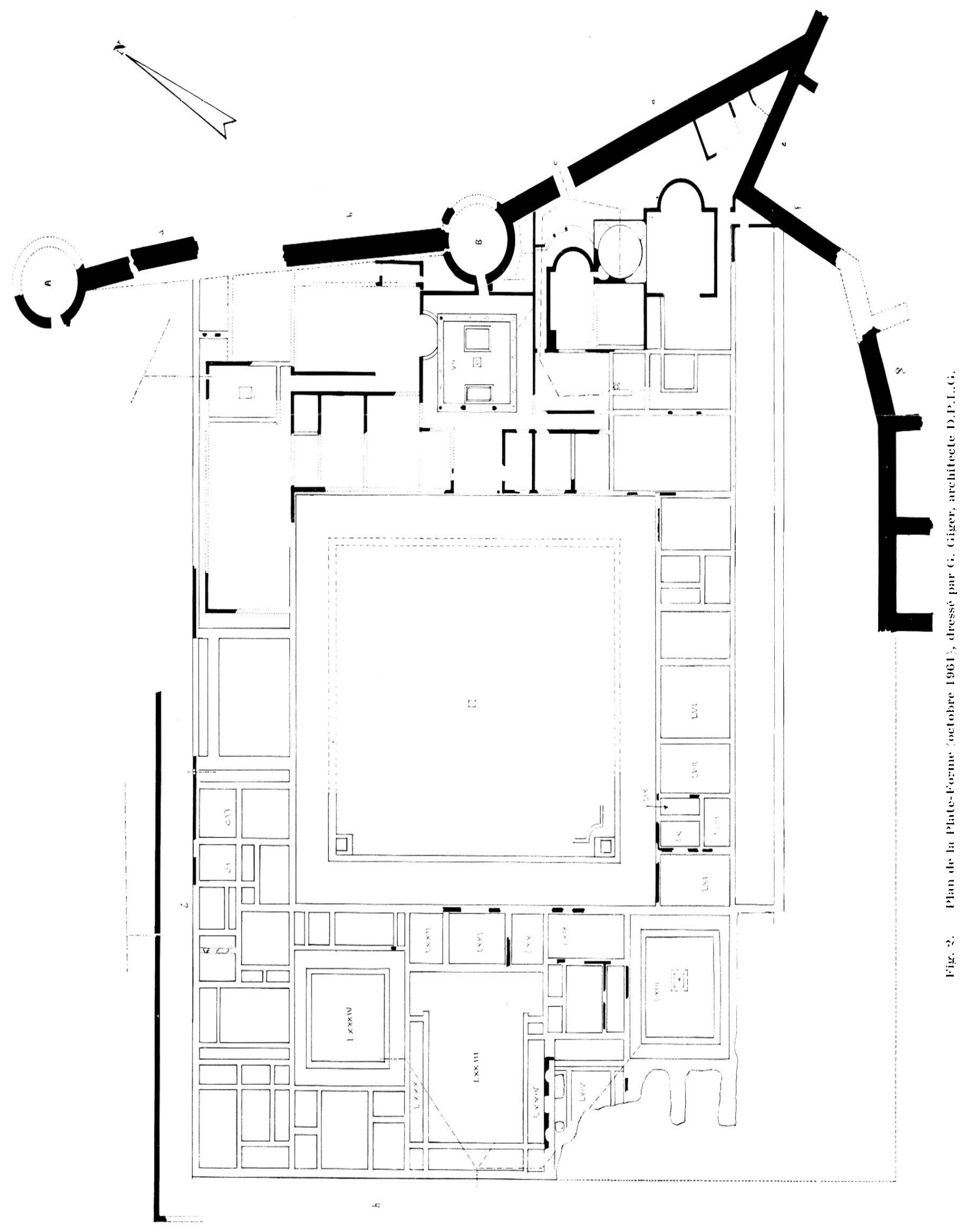


La muraille orientale qui délimite la Plate-Forme est l'enceinte urbaine que renforent deux tours rondes placées a 52 metres de distance l'une de l'autre, d'axe en axe. Diamètre des tours: $6 \mathrm{~m} .80$. L'épaisseur des murs des lours est de $1 \mathrm{~m}$. 1?, alors que l'enceinte est large de ? m. 35. Les tours s'ourraient vers la ville par une porte large de $1 \mathrm{~m}$. 50. Ine poterne était ménagée à l'origine sur cette face, au sud de la tour méridionale. Elle était large de $2 \mathrm{~m}$. 35. L'ouverture a été bouchée, soit lors de la construction des salles du terreplein, soit plus tard ( lig. 3 et 4).

Celte muraille a mal résisté à la poussée des terres et les tours aux parois relativement minces ont cédé. Leurs murs vers l'extérieur sont ouverts. De la mème façon le mur entre les tours s'est déversé. Cette ruine s'explique par le fait que l'enceinte n'a pas été fondée sur le rocher ; elle était placee au sommet d'une pente raide qui dominait un vallon. Ce vallon constituait une défense naturelle. mais le glissement des terres a entrainé le déchaussement des fondations. lies fondations. hantes d'environ 1 mètre (mesure prise a l'extérieur). sont faites de gros moellons irréguliers. Au-dessus commenee un mur en blocage avec parement régulier; les pierres sont de dimension assez forte ; les assises des murs établis sur le terre-plein seront plus minces. Détail particulier, sur certaines assises les pierres sont posées de champ ${ }^{6}$. A cette partie extérieure parementée, correspond vers l'intérieur un mur non parementé, ce qui fait penser que le talus de la butte primitive a été entaillé pour bâtir le mur. Deux trous de boulins qui gardent la trace de l'échafaudage se voient près de la tour méridionale (B); ils traversent le mur de part en part. La technique des tours est semblable. On note seulement qu'à la base de la tour A (celle du Sud (B) est trop détruite pour pouvoir ètre examinée). le mur s'élargit en talus. Cette semelle donnait une certaine stabilite sur un terrain peu solide.

Vers l'angle sud-est, les murs sont très ruinés. In glissement de terrain argileux a entrainé au bas de la pente raide une partie des constructions. Aussi est-il très difficile de faire dess restitutions. Ia muraille orientale (d) rejoint un mur (e) de mème technique qui fait avere elle un angle aigu. (Ce mur (e) épais de $1 \mathrm{~m}$. 8i) se prolongeait au-delà du point de rencontre, vers l'Est; il est rompu. Vers le sud, il est étayé par un contrefort large de $1 \mathrm{~m}$. 15) et long de deux mètres, qui a été rasé lorsque plus tard des constructions ont été faites à l'extérieur de la muraille. A ce mur (e) (voir plan fig. :2) fait suite un mur perpendi(culaire (f) large de $:$ m. (0). Ce tronģon de muraille est conservé en fondation sur une longueur de lis metres ; il s'est ensuite effondré. Jes bloes ont roulé le long de la pente, les fondations clles-mèmes sont disloquées (fig. i)).

A ce mur f, fait suite actuellement une muraille haule de 8 mitres tris bien conservée, g., dont les fondations reposent, non sur le sommet de la butle. mais au bas de celle-ci. Des contreforts rectangulaires qui étayent le mur, trois subsistent el il reste l'arrachement d'un qualrieme vers l'Est. In de ces contreforts a conservé sur sa face sud son parement vertical. Ces contreforts ne présentaient donc pas une face inclinée. comme ceux de l'aqueduc. Il semble cependant que de légers ressauts en diminuaient l'épaisseur vers le haul comme au mur de la ferme de la Ilorée priss d'Aix? Actueilemenl, au-dessous du petil appareil régulier ${ }^{8}$, est visible un mur plus grossier. qui devait èlre

(6) Iauteur des lits de haut en bas : $14, \overline{5} ; 14, \overline{5} ; 12, \overline{5} ; 19 ; 1.3 ; 11,5 ; 12 ; 15$. Épaisseur des joints : 2 a 1,5 cm.

(7) Carle arch. de la Gaule romaine, fasc. 1', Paris, 19:36, n" $: 13$, p. 59-60, fig. 3 .

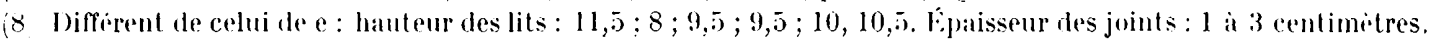




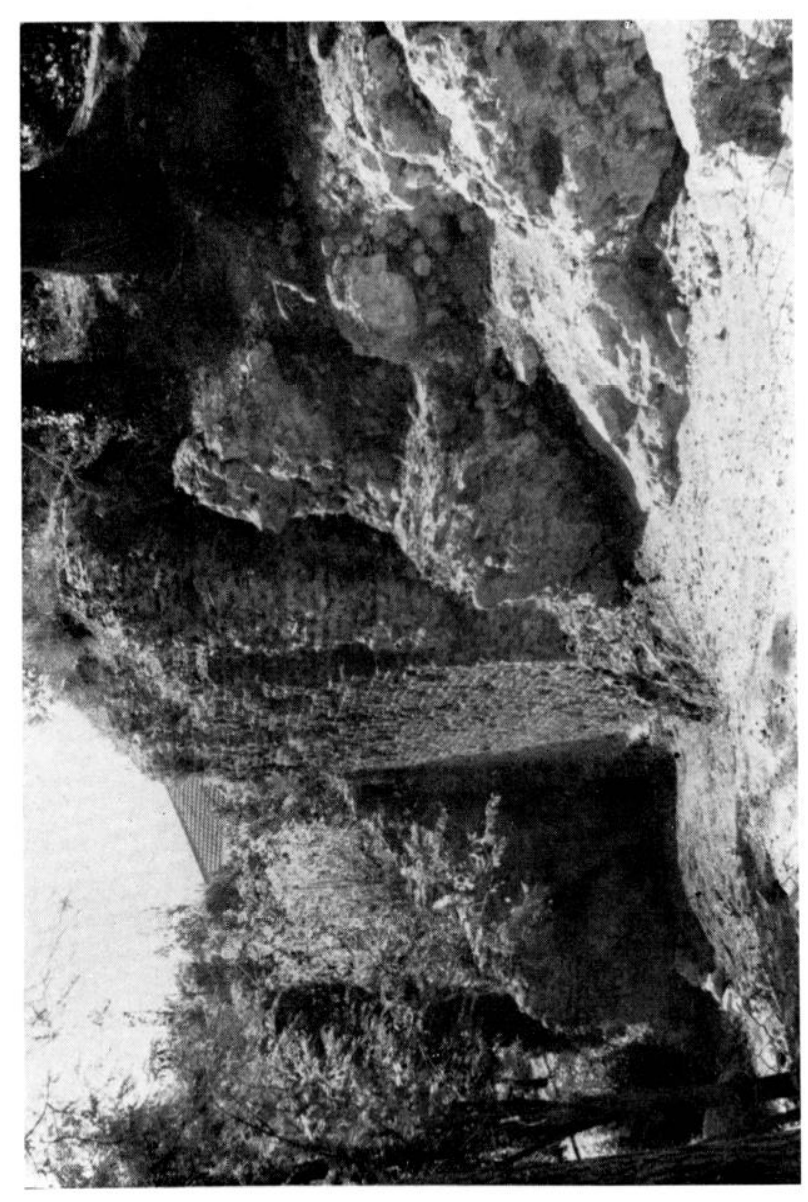

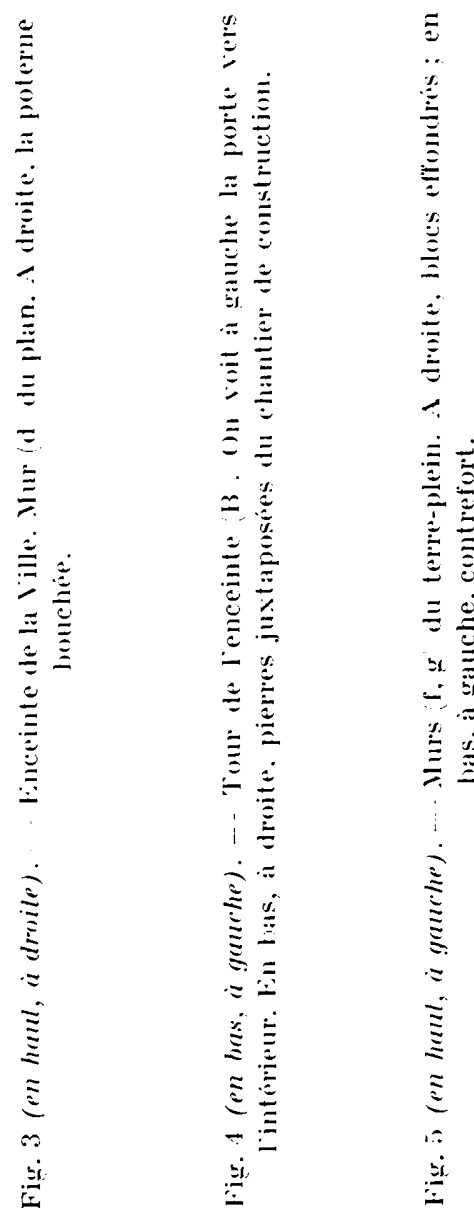
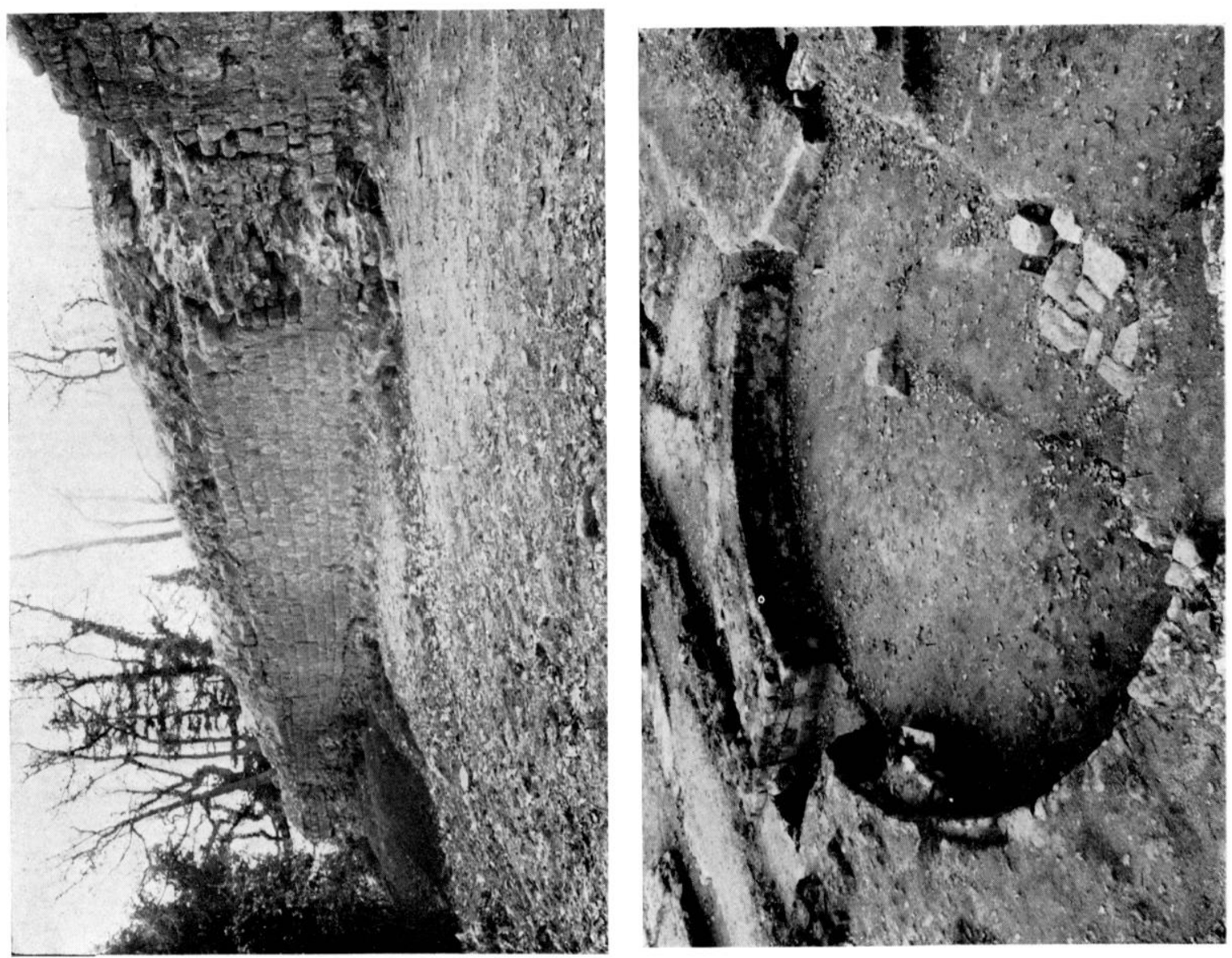
¿ l'origne dissimulé par un talus de lerre qui a disparu. Cescontreforts el la différence de lechnique montrent netlement un changement de parti entre les murs f et 2 . Y a-t-il une sensible dillérence d'àge entre les deux constructions? Jusqu'à présent, les fouilles n'ont pas permis de l'assurer.

I.e mur $\underline{a}$ est délruil vers l'ouest. L'effondrement est ancien rar il figure déja sur le plan d'Aubenas. El un dessin de .J. Quicheral 9 fait en $1843-1844$ montre que la ruine de l'angie sud-ouest présentait un aspect semblable à celui d'aujourd'hui. Mais acluellement toute chance de ret rouver le plan exact du mur a disparu car on a bâti au débul du siecle une grande villa (la villa Marie) lá ou devait passer la muraille. Il semble cependant possible de restiluer un mur droil qui allail rencontrer a angle droil la face olest du terre-plein.

Vers le sud-0uest, la dénivellation a racheter pour établir la Plate-Forme etait très forte, une huitaine de metres au moins. Aussi a-t-on fail le nivellement non en comblant,

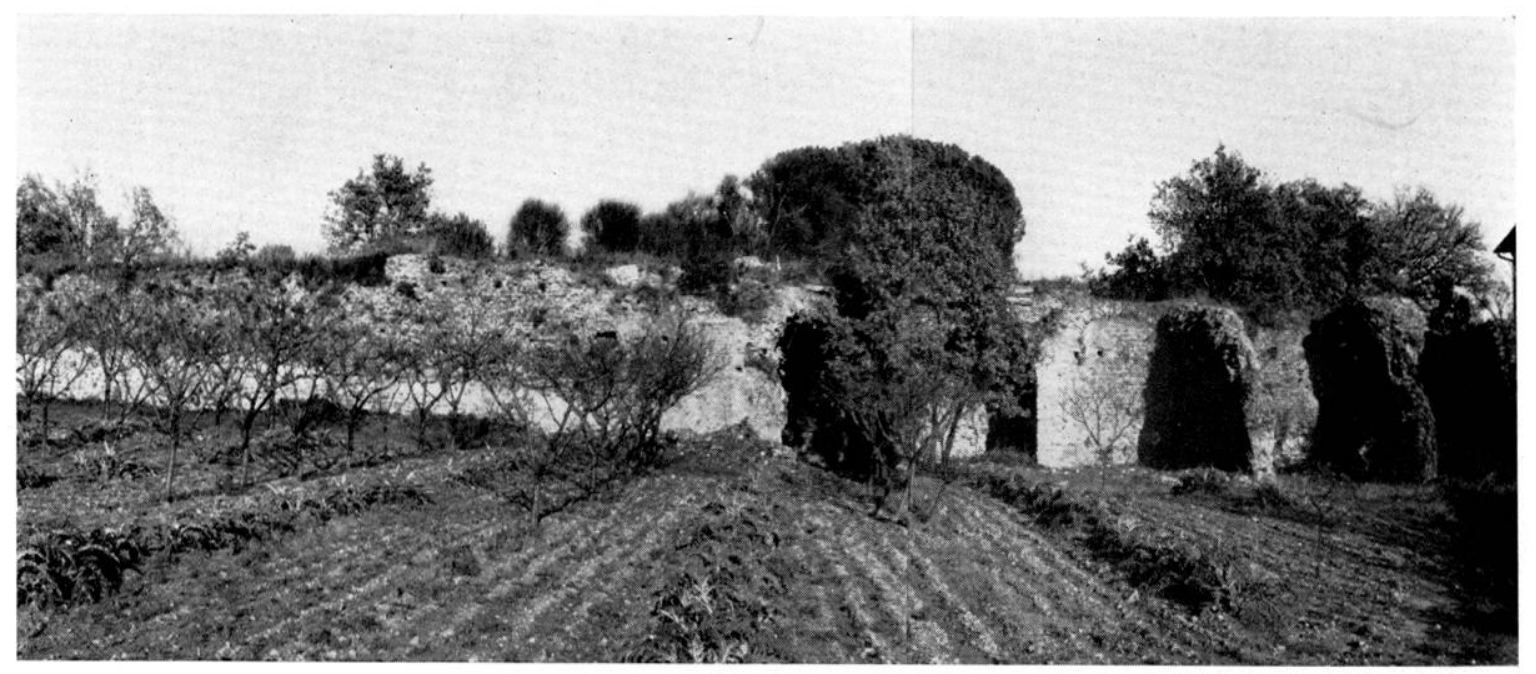

Fig. 6. Face occidentale de la Plate-Forme. A droite, les caves. A gauche, le mur plein en partie cache par les arbres.

mais en construisant de larges salles de profondeurs différentes, ch dont les voûtes supportent les constructions du terre-plein. Actuellement ring raves subsistent avec partie de leur voùte en plein cintre (fig. 6). Ies murs nord ot est d'une sixième so voient derrière la villa Marie. I 'examen du plan fait supposer une septième salle à l'angle sud-ouest. Ciertaines de ces raves communiquaient entre elles. Elles avaient vers l'Ouest des ouvertures d'environ 3 mètres de large. On voit encore en eflet un piedroit de celle qui est la plus au Xord. l)es fonilles ultérieures feront retrouver le plan de ce mur de façade. A cette partie fail suite, toujours sur la face occidentale un mur plein h dont le petit appareil régulier ressemble à celui de la face sud. Détail particulier, des intrusions de réticulé ou de pseudo-réticulé. (ar on a sourent utilisé des moellons allongés, qui dessinent des plaques de formes diverses. Fantaisies de maçons, sans doute, qui ont voulu montrer ou expérimenter ume lechnique qui devenait à la mode à l'époque augustéenne.

En deux points de la laçade distants de 8 m. 10, de part el d'aul re de l'égout figuré sur le plan. le parement presenle une coupure nelle sur plusieurs milles de haut. Or, en arriere de ce mur ouest,

(9) Paris, Bibl. de l'Écule des C:harles, J. (Guicherat, carnet 6. 
un sondage pratiqué dans l'espace LXXIIl el en LXXVIII a permis de retrouser un mur arasé

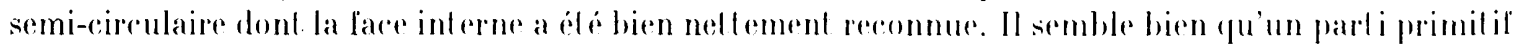
ait ése de ménager une exidere semi-circulaire vers le centre de reelle face, dans laxe des

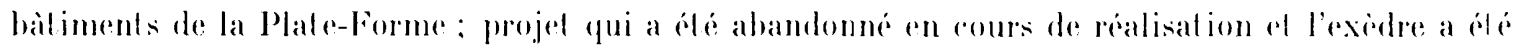

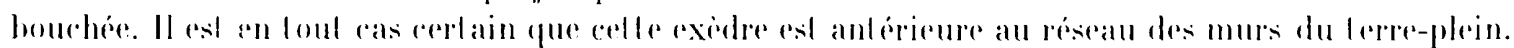

Au Norr, la muraille tourne a angle droit. Cetle face a été "n partie dégagée lors de la campagne de 1960 ( voir lig. ? ?).

Au centre de rette façarde que les fouilles du D D Donnadieu ont permis de restituer. est la porte monumentale. A l'bst de cette porte, le mur n'est. conservé qu'en fondation, sauf sur une courte partie, et le massif de fondalion alfleurail, avant les fonilles, presque le sol actuel. An niveau de la porte à l'extérieur du monument un sondage a ele fait audessous du mince mur de fagade épaisseur to centimetres) conserve sur quelques assises,

Jig. 7. Coupe nord-sud au revers du mur de facade Nord. A droile, le mur de facade; a ganche, le mur posteriemr. 1 : terre arable noire; : : sable fin avec quelques calloux:

3. calloutis compact; 1 : sable fin qui se mile vers le has

a l'argile; $\bar{j}$ : sable gramulenx; 6 : fosse de tranche remplie darerile rougeatre: 7 : argile verte plastique: $x$ : arrile ot sable tries compacts.

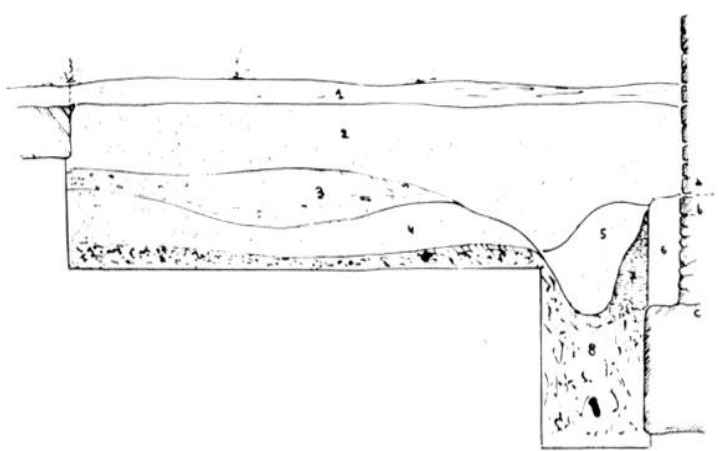

un mur d'appareil irréguliẹ sur une hauleur de $1 \mathrm{~m}$. 70; celui-ci repose, en retrait de 35) centimetres, sur un massif en blorage de 1 metere de haut. Ces fondations s'appuient

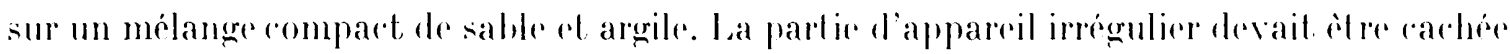

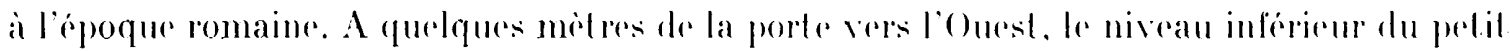
appareil reguliers sabaisse lapidement d'environ 1 metre, ce qui l rahit une forte déniverlation a l'origine. I'n sereond sondage (point i de la fig. ?) fait apparaitere que le mur de facade: repose sur un soubassement en petil appareil régulier qui s'est conservé en ere proint sur une hauteur de: 2 m. 10. Au-dessous, un appareil irrégulier sur 0 m. 60 de haut ; al plus bas un massif de blocage de 73 centimètres. Cette fondation repose la aussi sur le sol très dur d'argile et de sable fin mèlés. Ia fouille a permis de reconnaître qu'une tranchée de fonclation a elé crensée dans celte terre compacte. Sur une hauteur de 73 centimelres, était lassé un beton fait avere de petits éléments de gress. Toujours dans la tranchée, en rent rait de 30 cenlimelers a ete hati lo mur en appareil irrégulier jusquan nivean du sol ancien (lig. 7).

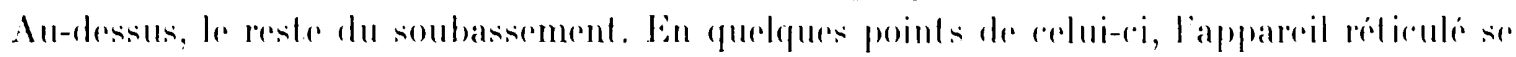
mèle an petit appareil régulier.

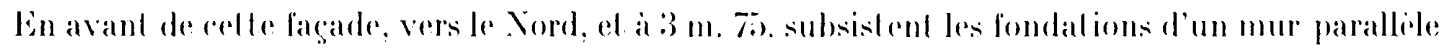
très ruiné. Ce mur qui se relourne au niveau de l'angle nord-ouest du monumenl pour suivere la fare oceidentale. parail bàti a une ćpoque ou déja une partie du soubassement de la Plate-Forme élail rachée par des apports nouveaux de lerre, ainsi que l'ont montré les deux coupes faites au niveau de k el de l (coupe fig. 7). Sur ce mur, au-dessus du niveau des fondat ions un bourrelet de ciment permet, de reconnaitre l'aspect du sol au moment de sa construction. Ge profil est diftérent de celui de la 
façade. Ainsi la dénivellation qui élait sensible à l'origine a été atténuée el c'esl une pente assez régulière qui devait faire descendre alors ce sol d'Est en Ouest.

Il apparait done que les murs nord-ouest et sud sont différents de ceux de l'enceinte urbaine : leur régularité dans le plan, leur technique le montrent bien ; leur orientation correspond à celle des monuments établis à la surface du terre-plein.

\section{La surface de la Plale-Forme.}

Sur la Plate-Forme ainsi délimitée. des bâtiments s'ordonnent autour d'une cour centrale, à laquelle on accédait par un large passage ménagé dans la facade nord. Lus mesures de la cour al niveau des fondations sont : au Nord $41 \mathrm{~m}$. 50) au Sud $41 \mathrm{~m}$. 30) à l'Est $44 \mathrm{~m}$. 75. à l'Ouest $44 \mathrm{~m}$. 80, soit sensiblement en élévation une cour de $41 \mathrm{~m}$. 5) sur 45 mètres. Tout autour à $1 \mathrm{~m}$. Tis des murs de la cour. un massif de fondation dessine un rectangle plus petit aux quatre angles duquel sont creusés de petits bassins rectangulaires. Cette fondation pourrait ètre la base d'un portique qui a disparu sans laisser de trace. La rour est actuell ment seuk ment à moitié dégagée et l'on peut espérer trouver quelque vestige vers l'Ouest. Deux des bassins ont été fouillés; celui de l'angle sud-ouest a $1 \mathrm{~m}$. $33 \mathrm{sur}$ $1 \mathrm{~m} .66$ et il est profond de $1 \mathrm{~m} .60$ environ. Les parois sont revètues de béton de tuileau; Ie fond est pavé de petites briques posées de champ en chevrons ; elles ont 2 centimetres sur 8.5. Cne ouverture de section triangulaire sur la face nord doit ĉtre une arrivée d'eau; mais il n'y a pas de trou d'écoulement. A quelques distance vers le Nord-Est un muret dessine un arrondi, sans doute est-ce là un reste du décor de la cour. d'un jardin peut-itre. Au centre de cette cour est la citerne foullée au début du siècle dernier.

Tout autour de cette cour. des bàtiments dont les pièces donnent soit sur la cour, soit sur d'autres espaces ouverts plus petits. Ene grande difficulté dans l'interprétation du plan est due à la destruction du monument dont il ne subsiste le plus souvent que les fondations. Rares sont les murs en élévation et ils ne sont conservés que sur deux ou trois assises au maximum. A noter la symétric de l'édifice par rapport aux axes Est-Ouest et Nord-Sud. La cour à péristyle de l'Est est dans l'axe de la cour centrale et du grand espace sans doute a ciel ouvert placé à l'Ouest (LAXIII). Les pièces de la partie sud sont symétriquement disposées par rapport à une pièce centrale.

En venant de la ville on entrail dans la cour par une large pièce rectangulaire. Le plan donné par le I) Donnadieu lors du Congries archéologique de $1932^{10}$ place à l'entrée quatre colonnes. Je n'en vois plus aucune trace maintenant. Cette disposition n'est pas invraisemblable car elle se retrouve au centre de la face est.

Lin effel. de ce còté. on passait de la cour dans une salle large de $5 \mathrm{~m} .88$ pour gagner une cour a péristyle autour de laquelle s'ordonnaient diverses salles. A l'entrée, sur l'alignement du mur de façade. l'empreinte d'une colonne de briques à 8 segments. de 45 centimètres de diamètre, est bien visible à droite. Aucune autre empreinte n'est conservée. Y avait-il seulement deux colonnes de part et d'autre ou un portique de quatre colonnes? Le passage s'ourrait donc largement vers l'Ouest. Il en allait peul-ìt e de mème vers l'Esıl où il n'y a pas trace de mur en élévation. La pièce était encore partiellement pavée, au moment de sa découverte, de plaques de marbre avec autour une bordure 
de mosaïques. Actuellement, on relève seulement des empreinles de plaques carrées sur un béton de tuileau et un fragment de mosaïque blanche à cubes très fins ${ }^{11}$.

La cour forme un carré (mesures intérieures : $13 \mathrm{~m}$. 60, couloirs compris ; $8 \mathrm{~m} .90$, couloirs non compris). A l'intérieur une galerie, large d'environ $1 \mathrm{~m} .70$, en borde les quat re còtés. Son sol en béton est partiellement conservé. Cette galerie donnait sur un espace a ciel ouvert par une colonnade en brique. Deux des colonnes sont en place aux angles du ròté nord ; elles ont 36 centimètres de diamètre et sont formées par six ségments de briques. L'empreinte des six colonnes de la face sud est nette at cela donne un entrecolonnement de $1 \mathrm{~m}$. 90. Vers l'Est, des colonnes ont aussi laissé leur empreinte fon en compte six sur

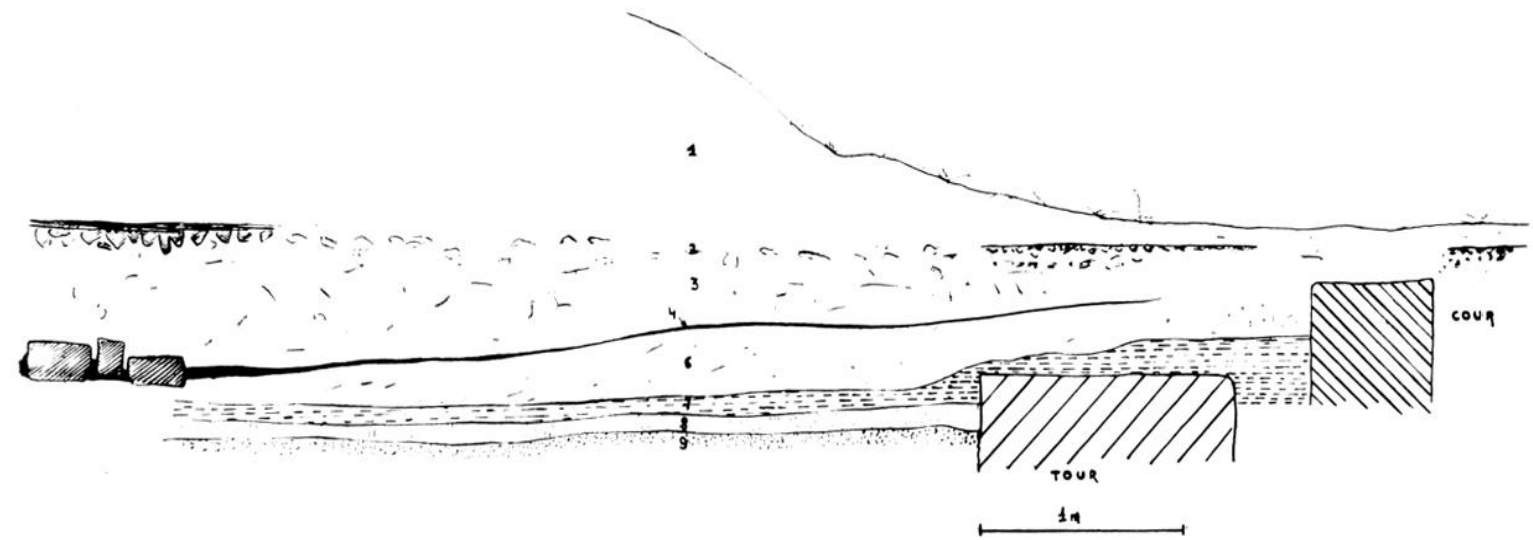

Figr. ‥ - (onpe list-()uest a travers la Tour B. 1 : Remblais modernes : 2 : sol et traces de héton marquant le niveau du sol : 3 : terre pougeatre : 4 : fine conche charbonneuse ; 6 : sable et gravier ; 7 : argile verte ; 8 : sable et argile; ! : sable.

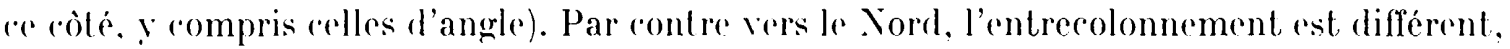
sans doute à cause d'un perron semi-circulaire qui parait postérieur à la cour. A l'ouest. de part el d'autre de l'axe de la cour, les colonnes sont remplacées par deux piles rectangulaires accostées de demi-colonnes. Dans l'espace central, un bassin rectangulaire pavé de carreaux de marbre blanc at de schistes noirs ; la tuyauterie de plomb a été arrachée. Plus à l'Est, un puits (ou citerne) et un bassin en béton de tuileau a bords arrondis. L'évaruation de l'eau se fait par un égout qui se dirige vers le Sud-Est.

A l'Est de cette cour. la tour de l'enceinte. In passage étroit fait communiquer la tour et la cour. En cre point. il a été possible d'étudier la chronologie relative des deux monuments. Dès l'origine. un passage arait été ménagé dans la tour. La base de cette ouverture correspondait sensiblement au niveau des fondations (fig. 8). Or ce passage a été partiellement obstrué à la base lors de la construction des fondations du mur oriental ; celles-ci dépassent le niveau primitif de 43 centimètres et le sol en béton de la galerie a été placé 20 centimètres plus liaut. Le niveau de la tour a donc été surélevé d'environ 60 centimetres. Sous ce sol de la galerie qui se prolongeait dans la tour, plusieurs couches de terre

(11) Cette partie orientale de la hutte a di en grande partie digagée par le I)r Domnadieu. Jintre 1939 et 1960 , le terrain a di ahandonne et des parties dicomvertes ont été tries ahimes par la pousse des arbres el des destructions. l.e site a été coupé par des tranchées. 


$$
\begin{aligned}
& = \\
& =2 \\
& z
\end{aligned}
$$


étaient visibles dont le dépôt était postérieur à la construction de la tour puisqu'elles passaient au-dessus des fondations de la porte. Au-dessus d'une couche de sable (couche 9), d'un mélange d'argile et de sable très compact (couche 8) qui ont été c'oupés par les fondations de la tour, reposait une argile verdatre qui a recouvert les fondations de la porte et parait bien avoir glissé d'Ouest en Est (couche 7). Puis vient une terre sableuse rougeâtre avec de petits éléments de gravier (couche 6). In filet de terre(couche"4) noire charbonneuse recourrait par toute la tour cette couche 6, et vers le Nord un tas de gravas (couche 5)). Cette terre noire formait 'n plusieurs points des poches où se mèlaient des éléments dr machefer. Près d'une de cess poches, deux pierres posées l'une sur l'autre, ayant sans doute appartenu a un foyer. En contact aver cette couche 4, vers l'Est un lit de pierres en cours de taille, parmi lesquelles quelques moellons (face taillée: : 3: sur 14 et 99 sur 11) : trace sans aucun doute d'un atelier. Vers l'Ouest et toujours au mime niveau, trois plaques de marbre de décoration. Étant donné que cette couche 4 passe bien au-dessus du seuil primitif, on ne peut supposer que cet atelier est celui de la tour; on pensera plutòt à celui de la cour. Au-dessus, une terre rougeàtre (rouche 3). Ie sol s'est conservé particllement près de la porte $(2 a)$ et plus à l'Est. Entre ces deux points une différence de niveau de 14 centimètres.

sous ces sols, quelques éléments de céramique fournissent des indiees de chronologie ( lig. 9).

Couche 4 : 1. Fragment de coupe à pàte ocre micarée. décorée a l'extérieur de cherrons, engobe brunitre i l'exlérieur, rouge is l'intérieur. peu adhèrent (ins. PF 177.1;

2. Fragment de col i patte ocre clair ; engobe rouge $(1803)$;

3. Col d'amphore a pate jaunatre (1679) ;

4. Col d'amphore a pate jaunàtre fine (1709 et 1735) ;

$\therefore$. Rebord de céramique commune $(1707)$.

Couche 3 : 6. Fragment de rebord d'Arezzo, service I de Hallern (1532) ${ }^{13}$;

7. Rebord de grand plat, céramique commune orangée, micacée (1407);

8. Fragments de bol a paile orangée trìs bien cuile. Vernis extérieur réservé sur la partie supérieure; vernis intérieur orangé aver bande plus sombre au sommel. De tradition campanienne $14(1526-1531)$.

9. Rebord de pâte lendre ocre, micacée. Vernis peu adhérent brunàtre, de tradilion campanienne (1614);

10. Large col de cruchon. pot erie grise (1490);

11. Col pàte ocre (1452);

12. Col étroit pâte grise (I674).

Couche 2: 13. Fond de plat d'Are\%o (1736);

1.4. Rebord pot erie commune ocre (1730);

1:) a 18. Rebords poterie commune, amphores (1.11:, 1:5i1. 17.47);

19. Rebord de grand plat (1.480);

Provenant sans doute de la couche 3 (sondage préliminaire):

20. Anse de lampe à pâte ocre, vernis mal noir (137i);

21. Petit vase à incisions (1388).

(12) Ce materiel est actuellement conserve an centre de Documentation areheologgigue du lar a Dlagnignan. Il est inventorié sous le sigle PF Plate-forme avec un $n^{\circ}$ correspondant an journal de fouille.

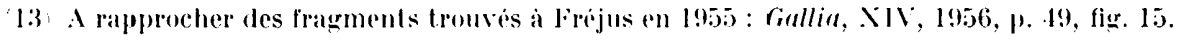

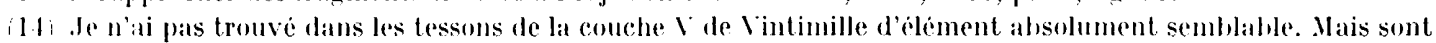

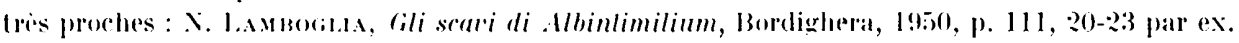


FOLILLES IDE FREJTS

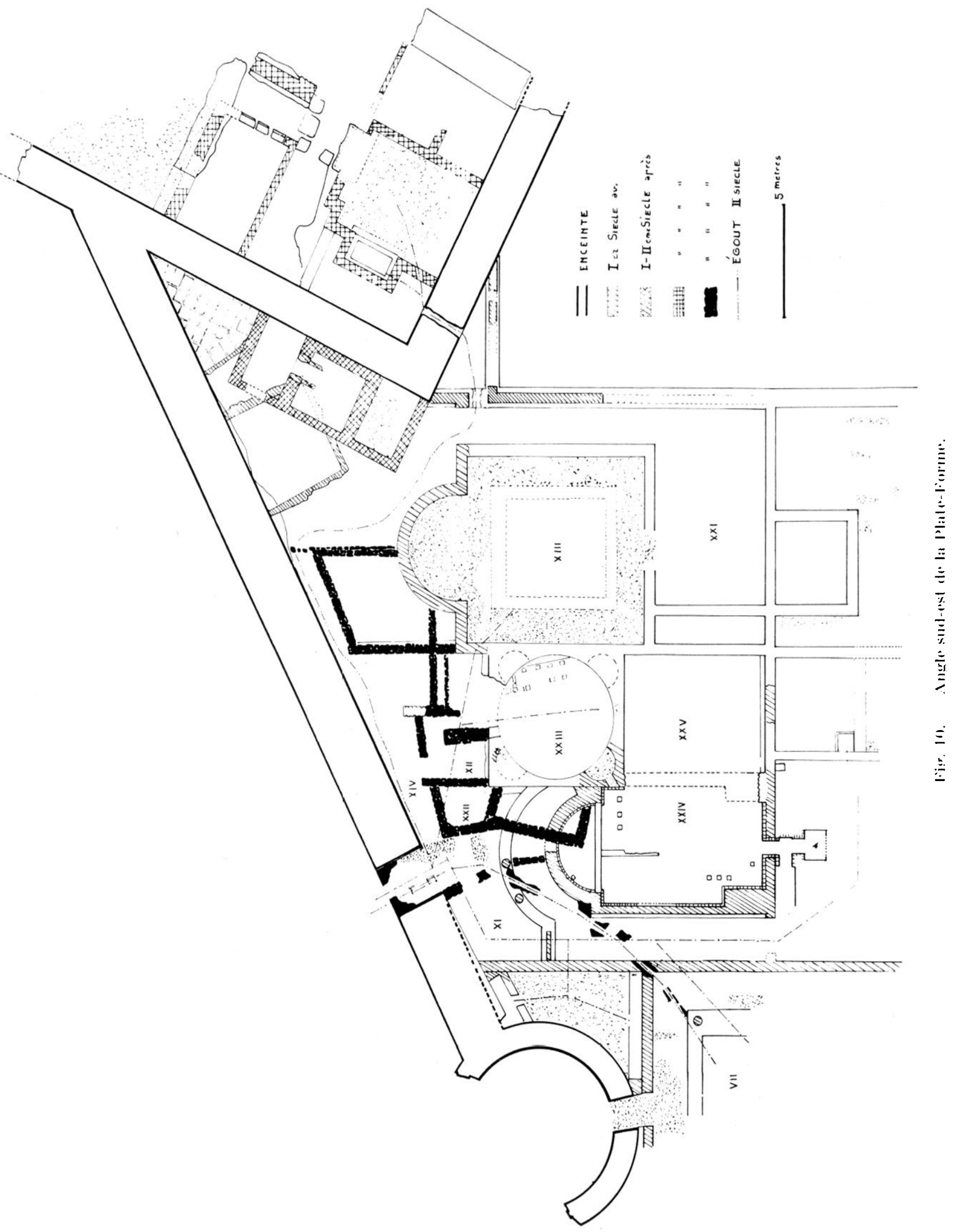


Ce matériel est relativement pauvre. Mais il est comparable à celui de la couche $V$ de Vintimille. Il apporte un intéressant document pour la clironologie de la Plate-Forme et celle de l'enceinte.

Dans la galerie nord du péristyle, une large porte ourre sur une vaste salle rectangulaire (VII) qui a conservé son pavement sur une large surface. I.e béton lissé est semé de fragments irréguliers de marbres blanes et noirs disposés en files perpendiculaires les unes aux autres. L'entrée a été remaniéc ; une exèdre semi-circulaire a alors été construite dans la galerie. A l'Ouest de cette pièce, un couloir large de $1 \mathrm{~m}$. 45) sépare la grande salle de pièces plus petites qui ont gardé elles aussi un sol de béton avec incrustation de fragments de marbre. Ine autre pièce (I) avait un sol fait d'un mortier peu résistant avec au centre une petite piscine rectangulaire de $1 \mathrm{~m}$. 60 sur environ $1 \mathrm{~m}$. 10. Ce sol a été en grande partie détruit. Vers le Nord un petit égout amenait les eaux vers l'extérieur de l'édifice et de là dans l'égout parallèle à la façade (fig. ?).

De l'angle sud-ouest du péristyle part un couloir Nord-Sud, qui a conservé une grande partie de son béton. Il mène à diverses salles où sont installés des thermes. Il est tròs difficile d'expliquer certaines parties du plan car le monument a été très endommagé dès l'époque romaine, a subi des remaniements et des destructions semblant être intervenues depuis l'époque du Dr. Ionnadicu. En outre, ce dernier avait partiellement fouillé lo monument et je n'ai trouvé trace des remaniements que dans les parties où il n'avait pas travaillé. Aussi est-il impossible de préciser exactement l'extension de ces transformations.

L'état primitif des thermes peut ètre ainsi décrit: au Nord, une salle à abside (XXIV). séparée de la cour par un couloir qui suit un égout; au centre une salle ronde (XXIII); au Sud une salle à abside (XIII), enfin à còté diverses pièces (fig. 10).

Le couloir XI isole la salle XXIV de la cour ; il se prolonge autour de son abside par un promenoir qui s'ouvrait vers l'kst par une colonnade. Deux fûts de colonnes en briques (diamèt re $0 \mathrm{~m}$. 36) sur quatre ou cinq subsistent. La salle ì abside élait une salle chauffée ; son sol inférieur subsiste en srande partie; les murs Nord, Est et Ouest sont encore revilus de briquettes (20/12, 5/3, 5), et des pilettes carrées subsistent en quatre endroits. La salle de chauffe devait it re alors en XXVI : en effet une ouverture sous un arc est ménagée dans le mur occidental de XXIV; les parois sont couvertes de briquettes et un sondage en sous-muvre, sous les mosaïques alteste la présence d'un couloir de chauffe (voir plan fig. 10, a). Le mur sud de la salle à abside a entièrement disparu ; le sol inférieur même a été emporté. Les arrachements de ce mur laissent supposer que la salle devait communiquer avec la piece voisine XXV, au moins au niveau des hypocaustes (voir fig. 10 et 14). De cetle salle XXV rien ne subsiste que les murs.

En XXV, aucune trace du sol au-dessus des hypocaustes ne subsiste. son niveau est seulement assuré grâce au sol de béton conservé dans une niche de la salle ronde XXIlI. là où ét ait une porte de communication. En XXIII. une pièce ronde inscrite dans un massif carré aux quatre angles duquel élaient logées des niches légèrement oulrepassées: une seule, au Nord-(ouest, est bien conservée. Des traces subsistent a peine a l'Est. Au centre des faces est et ouest. quelques fragments de béton de tuileau font supposer en des points la présence de portes qui ont disparu totalement. Donnadieu avail signalé dans ces niches des mosaïques blanches et noires qui onl elles aussi disparu. J'en ai seulement trouvé des débris dans le centre de la pièce. Lin contrebas de 3i) centimètres par rapport au niveau de ces niches. le niveau inféricur de l'hypocauste avec quelques pilettes (21/22 ou 22/23; ép. 3. 2) en place. A l'kst. reste de l'ouverture du praefurnium et un canal pour évacuer l'eau. 

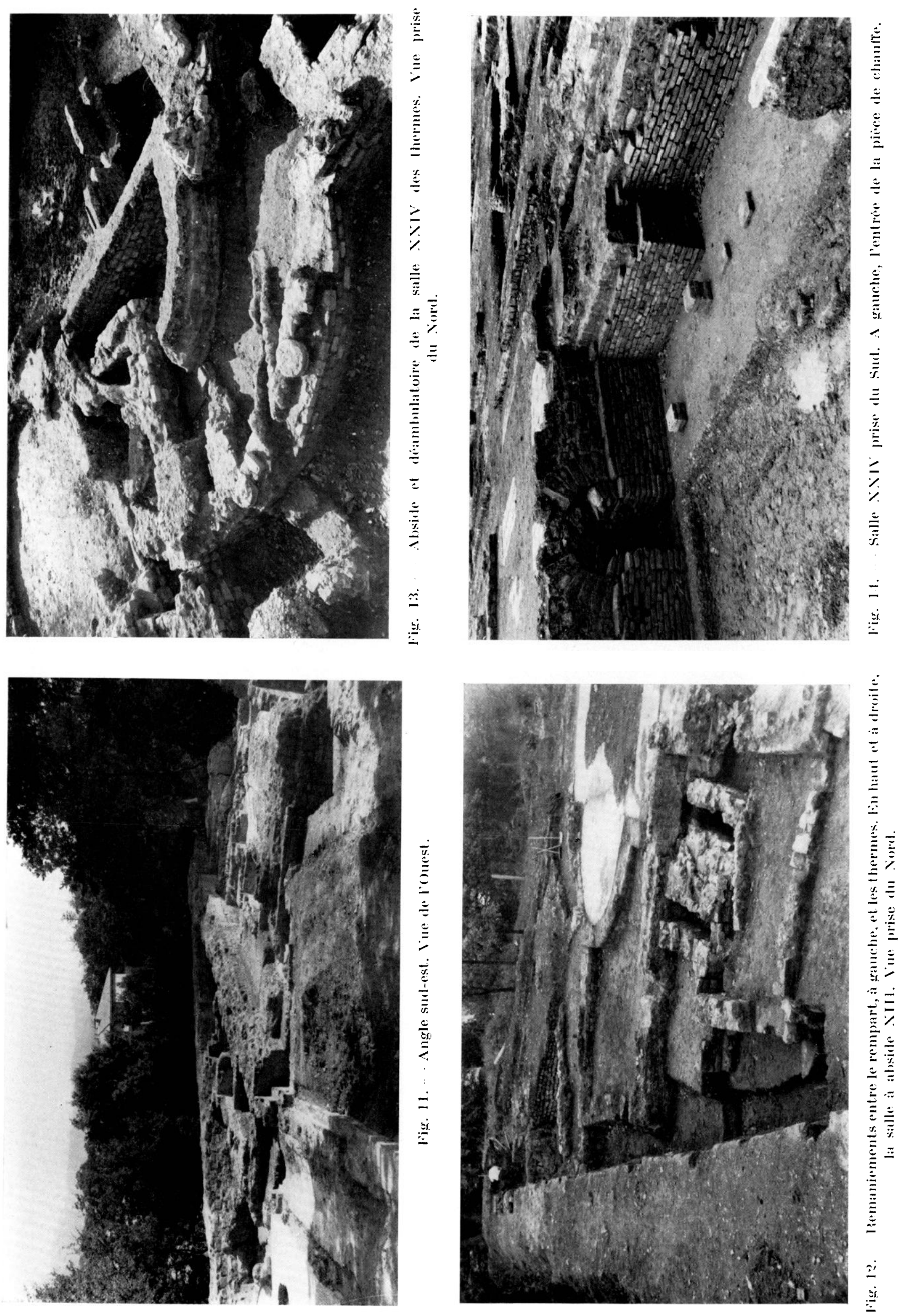

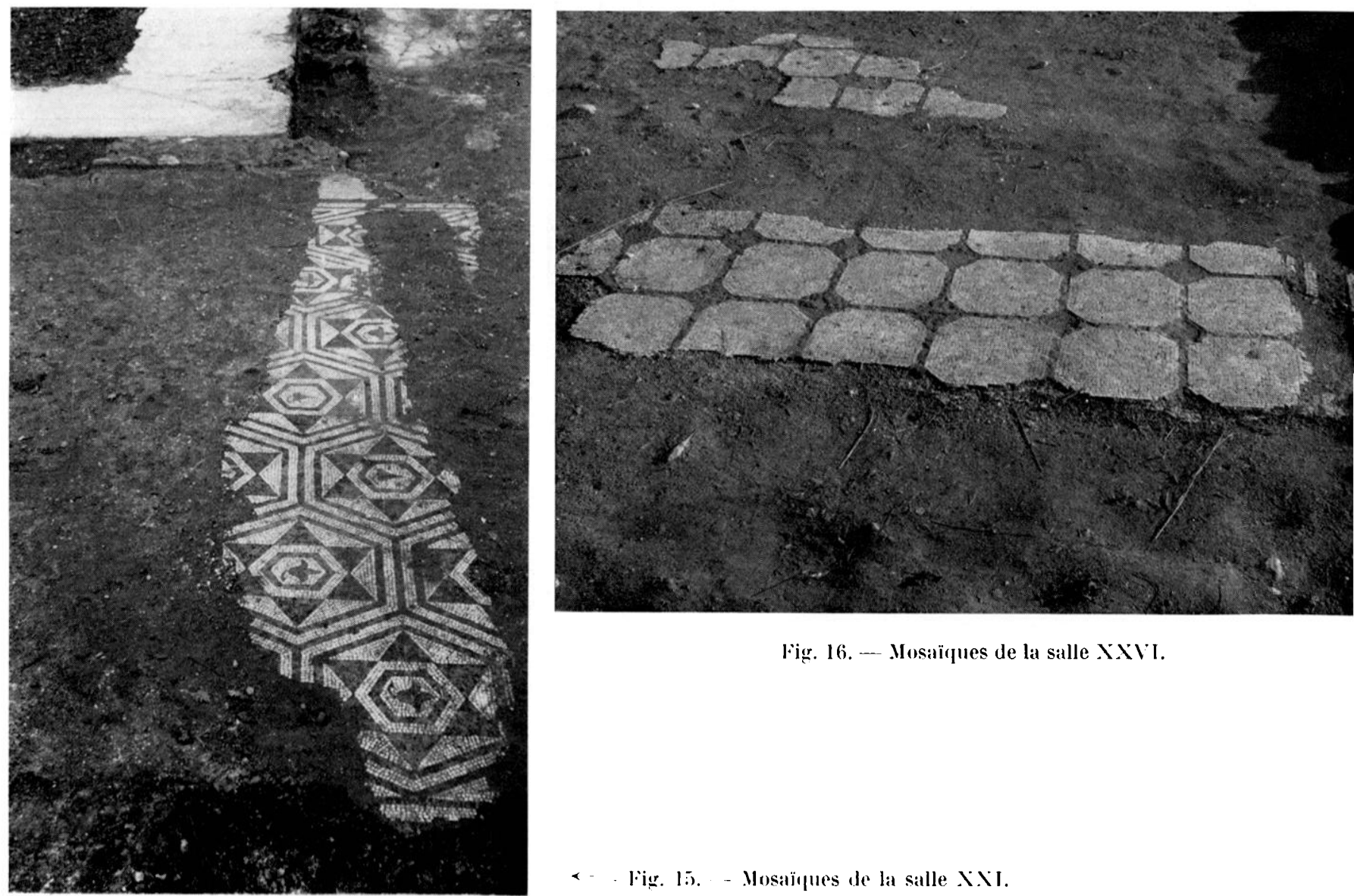

Fig. 16. - Mosaïques de la salle XXVI.

Iu Sud, la salle a abside qui a gardé son bélon armé lissé sur tout le pourtour. Au centre. il devait y aroir une piscine peu profonde (l'argrile vierge apparaîl en place à 60 centimètres au-dessus du sol conservé). Des empreintes d'un emmarchement apparaissent contre le béton. Celle salle longée vers le Sud par un couloir qui a conservé son sol. De ce couloir on passe dans l'espace non bàli qui s'élend partout au sud des bâliments de la Plate-Forme. Vers l'Ouest la pièce XIII ouvrait sur la salle XXII : des fragments d'un béton de tuileau sur le mur de séparation indiquent l'existenre d'une porte. La pièce a conservé une partie de son pavement à mosaïques blanches et noires. Sur fond blanc. des étoiles a six branches inscrites dans des hexagones de 3í centimètres de cóté. Au milieu de chaque eloile. une feuille (fig. 15). Tout autour de la piece, des bandes blanches el noires.

Il est possible que les pieces XXII. XXIX. XIL. XLVI. XI.VIl el XIVIII aienl dépendu de res thermes. En XXIX se voit un pet hassin de 79 sur 8:3 centimetres, peu profond (environ 75 au-dessous du sol présumé), d'où part un égoul qui l raverse la salle XXVI pour passer dans le couloil entre la cour à péristyle et XXIV. Ces thermes comportaient donc deux salles chaulfées au moins, donl une plus pelite que l'aulre al une salle froble au sude ainsi que diverses annexes. Mais l'blal de la ruine emperche d'itre plus précis.

Cot edifice primitif a ronnu des transformations. Signalons d'alorol relui de la sallr. XXVI. I a salle de chauffe a été détruite el remblayée. In sol nouveau a été établi avere une. mosaïque blanche décorée d'un quarlrillage de rubes noirs (lig. 16). Autour de la pièce. une hande noire larese. Je ne sais exartement a quel moment placer eette modifieation par rapport à celles qui se remarquent dans le reste des thermes. Elle doit seulement dater d'un moment où une partic au moins des thermes était ahandonnée. 


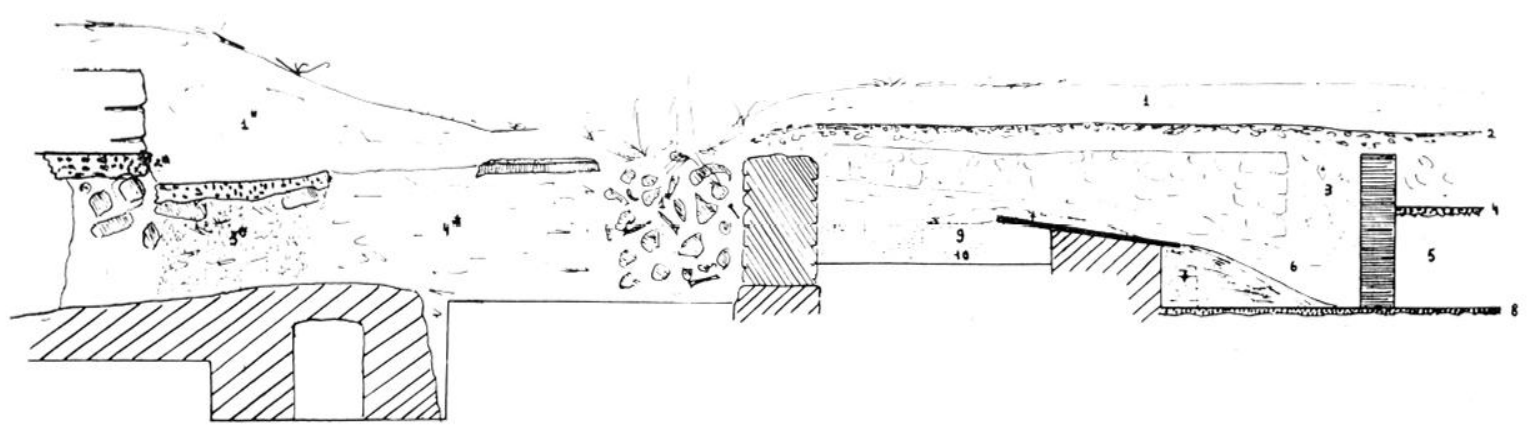

Fign. 17. Compe Est-Ounst an nivean de la poterne du rempart du dómbulatoire et de l'abside de XXIV.

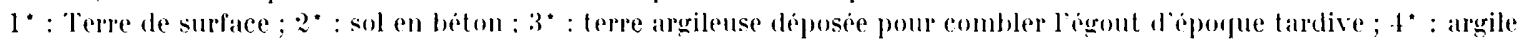
jallutre.

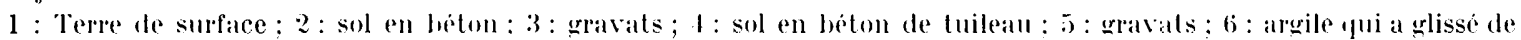
l'list ; $7:$ terre charbonmeuse $: x:$ belon de tuilean ; 9 : sable : 10 : arwile verte.

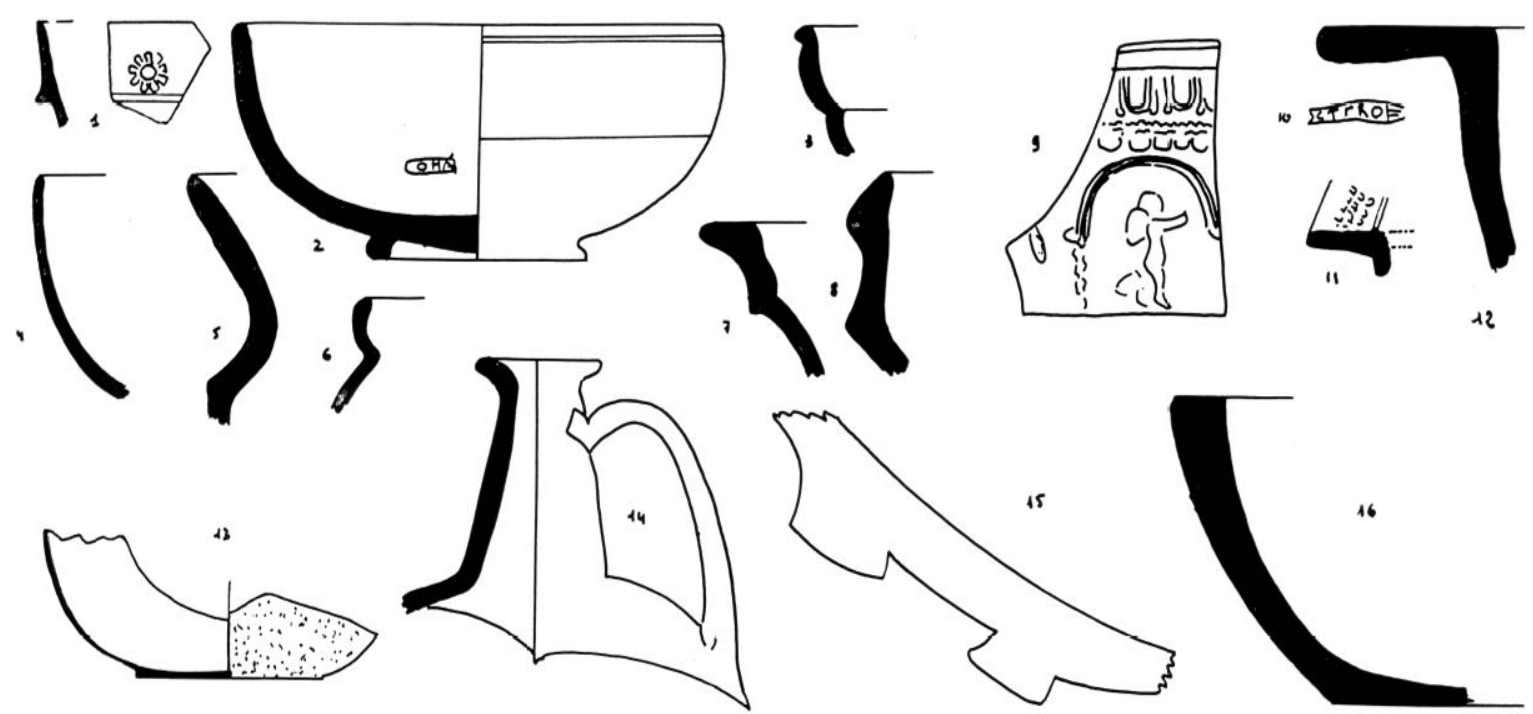

Fig. 18. .... Ceramique des salles XII et XXII.

Des murs irréguliers s'appuient sur ceux des thermes, à l'Est. lls leur sc nt postérieurs : au cours de la fouille, il a été reconnu qu'ils ont été bàtis à une époque où le promenoir qui entoure l'abside XXIT était ruiné, et où l'abside était aussi ruinée car ils enjambent ou remplacent des murs anciens. La question est de savoir si ces modifications se sont étendues au reste des thermes. les murs nouveaux ont été retrouvés sous un sol qui avait, arrèté la fouille de Donnadieu, sol qui s'étendait sur la partie nord de XXIV. sur le couloir $\mathrm{XI}$, et presque partout entre ces deux pièces et le mur d'enceinte. Tonc tout ou partie des thermes a élé transformé.

Ces pièces aux murs d'un appareil irrégulier où sont mèlés grès bruns et verts peuvent ètre datées approximativement. I’ne couche de terre brunàtre (voir fig. 17) qui s'est déposée. au-dessus de l'argile vierge et des égouts primitifs a été entièrement fouillée. En un point. il a été reconnu qu'elle élait au-dessous du sol de ces pièces et donc antérieure à ces murs ou leur contemporaine: un enduit blane subsiste sur un mur entre XII et XXII et donne le niviau. 
Voici le matériel trouvé dans cette couche:

Salle Xll (fig. 18) : 1. Fragmenl de rebord d'Arezo (2219, 2248);

2. Bol du sud de la (iaule. Forme Dragendorf 40. Marque O H . \ $(2256)^{15}$;

3. Rebord du sud de la (raule. Dray. $27(2250)$;

4. Rebord de céramique micacée, à engobe rougeatre; imilation locale de la sigillée (2277);

5. Rebord de grand vase. Céramique locale (2268);

6. 7. 8. Cols (2288, 2265, 2281);

9. Fragment d'Arezo (2314).
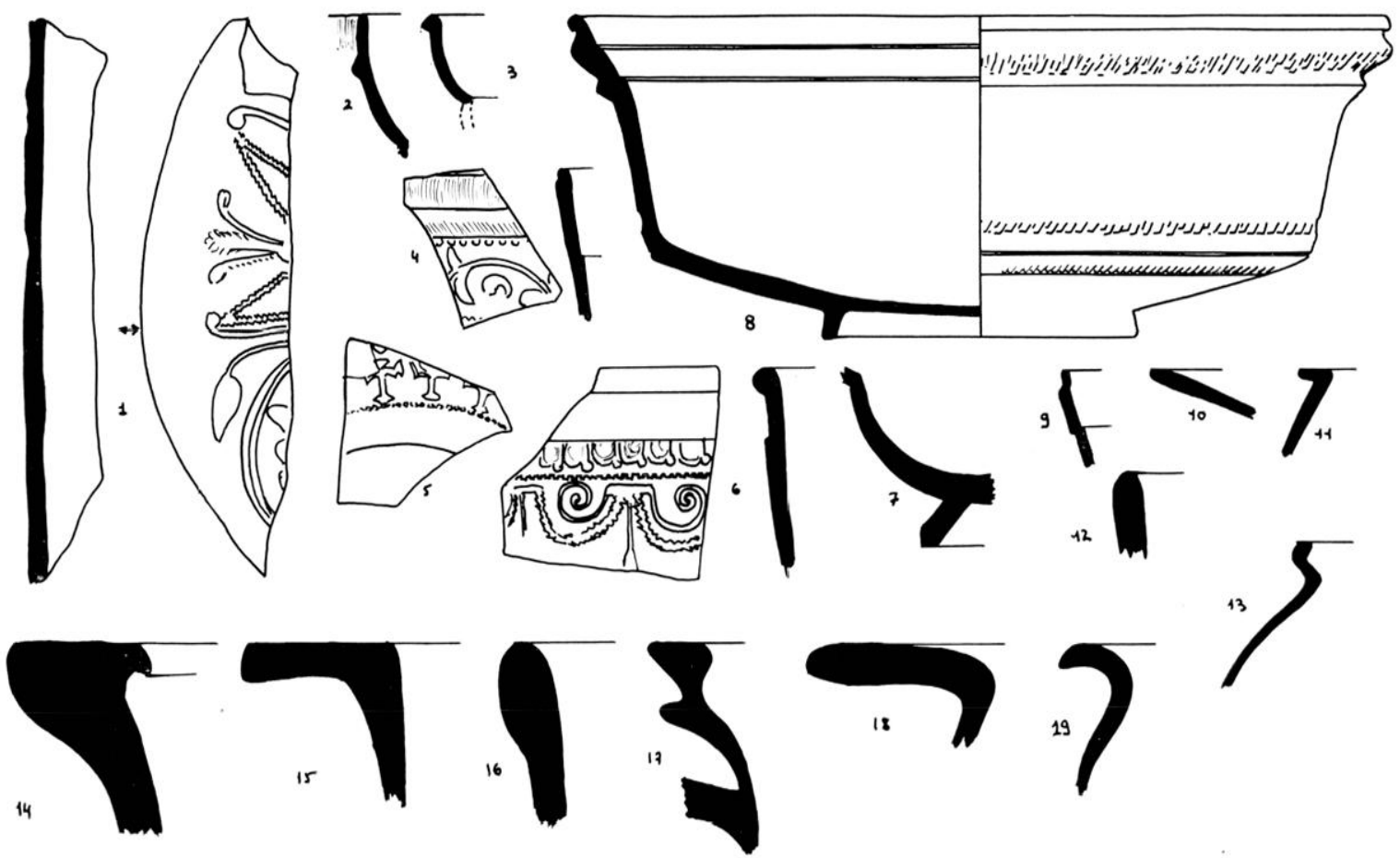

lïgr. 19. - címamique du fossé de l’égoul.

Salle XXII : 10. Fragment de fond d'Arezzo (2316). Marque OCT PRO in planla perdis. ${ }^{16}$;

11. Rebord de couvercle en sigillée claire $A$ (2301), forme $20^{17}$;

12. Rebord de grand vase de poterie commune (2:307).

Salle XXIV : 13. Fond de coupelle de poterie fine sablée (2426, 2427);

1.1. Col de cruche à pàle orangée (2418);

15. Fragment de grand plat de 40 centimitres de diametre avec deux oreillettes de préhension (2426, etc.). Polerie noircie it gros grains de calcite.

D'autre part. Le long de l'enceinte une fosse a eté creusée (courche $3^{*}$. fig. 17) pour recevoir au fond un petit égout en brique qui va se jeter dans la canalisation primitire. Ine des tuiles trouvée en place porte la marque CAstohIS18. Dans la terre qui remplissait la fosse ont élé trouvés de nombreux fragments de céramique (fig. 19).

(15) Rapprocher de la forme: F. Oswato et T. D. Prove, An Introduclion to the Sludy of Terra sigillala, Londres 1920, pl. XLIIII, 7 .

(16) C.I.L., I. I, $670045 \%$.

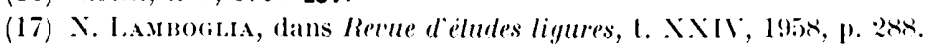

(18) C.I.L., t. XII, 5679 15. 
1. Fragment de cruche plate du sud de la Gaule avec décoration de médaillons ef de sautoirs $(1888)^{19}$ :

2. Rebord de coupelle du Sud de la (Gaule. I)rag. 24 (1988 el 1989) :

3. Rebord de coupelle du sud de la (iaule. Drag. $27(2016)$;

4 a 6. Fragments du sud de la Gaule (1891. 1893. 2018);

7. Sans doute forme Drag. $27(2017)$;

8. Grande coupe de sigillée claire $\Lambda$ (1931 a 2021$)$; forme Lamboglia I $\mathrm{B}^{20}$;

9. Rebord de sigillée claire, sans doute forme $7(1948)$;

10 et suivants, céramique locale : 2046, 2194, 1907, $1968 ; 2138 ; 1954$ (pâte ocre), 2032 (id.), 1902 (id. et engobe rouge). 1986 (pâte ocre).

In autre petit égout a été retrouvé partiellement, à l'angle de la cour VIII, contre les murs du promenoir à colonnarle, au niveau de l'ouverture qui était ménagée dans le rempart. I)ans la cour VIII où il avait été reconnu par Donnadieu, un des éléments qui épaulait sa paroi sud, était un fragment de brique avec l'estampille d'Hérennius ${ }^{21}$. Et dans la terre noire, sans doute non touchée par les anciennes fouilles, un fragment de sigillée claire A.

Cies murs irréguliers. et particllement les deux égouts précédents ont été recouverts de gravats avec de nombreux fragments de luyaux et. par-dessus, un sol de béton grossier et peu solide partiellement détruit. Il n'a pas été possible de reconnaître le ròle de ce sol ni de le rattacher à aucun des murs conservés, tant le terrain était bouleversé.

L'analyse du matériel montre que les salles XII et XXII sont postérieures au I er siècle, à cause de la découverte de fragments de céramique du Sud de la Gaule et d'un tesson de sigillée claire. La tranchéc est elle-mème datée par ce vase de sigillée claire A, forme I B. qui parait à Vintimille dans la seconde moitié du II $\mathbf{e}$ siècle. On notera qu'en mème temps que la sigillée claire A apparaissent les marques des potiers Hérennius et Castor. Or celui-ci a laissé sa marque sur une des briques de l'amphithéâtre ${ }^{22}$.

Avant de passer au reste de la Plate-Forme, je signalerai à l'extérieur des thermes les modifications qu'a connues l'angle sud-est de la butte. On distingue là plusieurs époques. mais la fouille ancienne a fait disparaitre tout élément de datation. Dans l'angle aigu des deux murs de l'enceinte, une pièce triangulaire (XX) était pavée de largıs briques. Le sol était recouvert de chaux éteinte, une autre pièce carrée (XYIII) était disposée de mème façon. Toutes deux ont été coupées par trois salles postéricures (XXIX-XXX-XXXI) : l'une était chauffée par hypocaustes (XXX). XXXI conserve un sol en béton de tuileau, plus haut que celui des thermes. Aucun indice ne permet de dater cette construction par rapport au reste de la plate-forme.

De façon étrange, les Romains ont aussi étendu leurs constructions au-delà de l'enreinte; sur la pente du terrain, il leur a fallu faire d'importants travaux de remblaiement. Malheureusement pour nous, car la plupart des murs se sont effondrés à la suite d'un glissement de terrain. On ne voit plus que deux salles dont une avec deux piscines (XXXVI). Je cette salle part l'égout qui va se glisser entre les thermes et l'enceinte el qui se troure

(19) Oswatid-PRYCE, op. cil., pl. I.XXXY, 1.

(20) X. I.AMBoglia, art. cil., p. :203. et 205 .

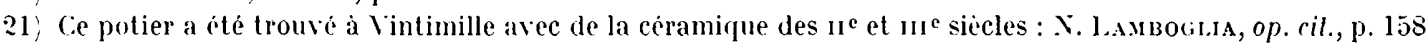

(2:) Photo dans Revue déludes ligures, t. XX11, 1956, p. 170, fig. 21. 
daté du I $^{\mathrm{e}}$ siècle. Il est impossible de restituer le plan exact de cotte partie. Je note seulement, au milieu des sols, des murs éboulés, des pilettes rondes d'hypocaustes et en XXXVII. l'ouverture d'un four qui a disparu. Peut-ètre y ent-il là des annexes des l loermes aménagés. au moment où les plus anciens furent remaniés ou détruits.

Dans le reste de la Plate-Forme. les problemess sont moins complexes rar on ne lrouve pas de trace de remaniements (sauf en LXXIII ot sans doute en L.XIV). Sur la fare noril. les pièes disposées symétriquement sont rasées jusqu'aux fondations, sauf vers lo(ouest. Lat on peut reconnaitre que l'espace étroit I.IX est un couloir ouvert sur la cour, que l'on passait de LXIII a L.XI par une porte, que I.X ne domnait pas sur la cour par une porte

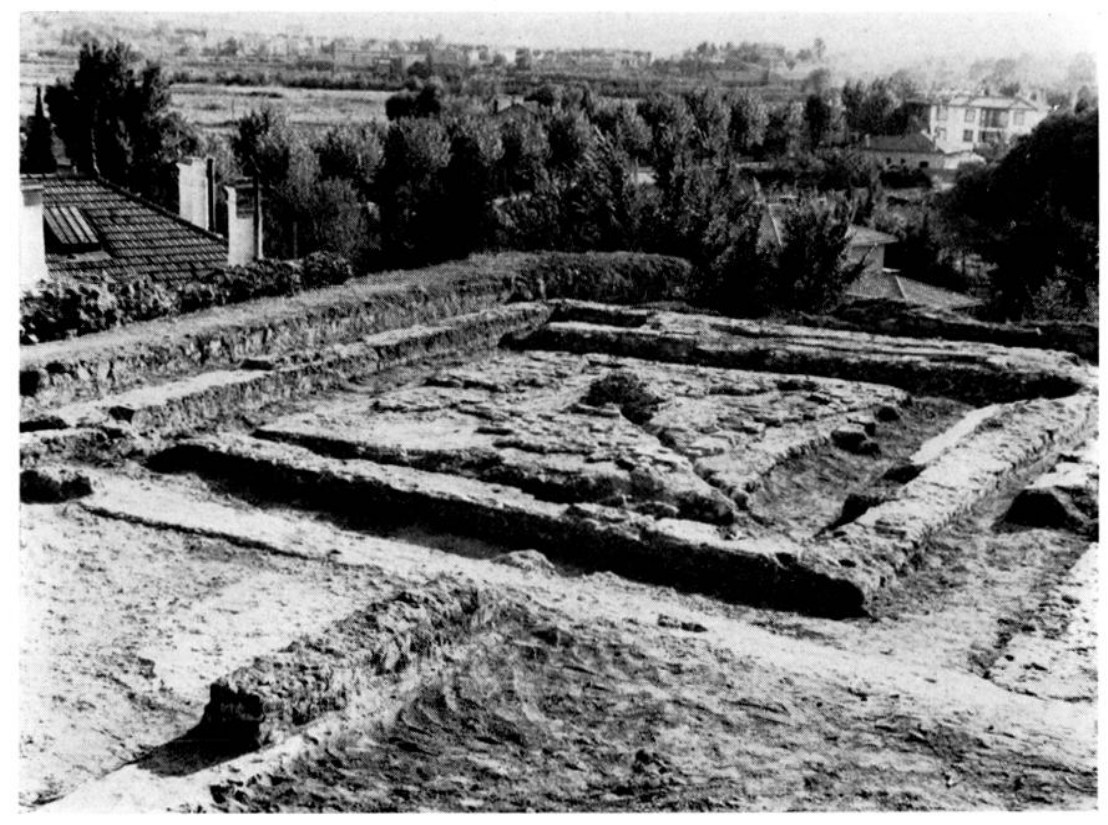

Figr. :0. Salle l.XIII vue du Vord-list avec son parement.

percée sur son axe. LYII a conservé un sol de béton. La salle LXI était richement ornér d'un parcment de marbre at de pierres de couleurs dont subsistent quelques éléments Ine sorte de marqueterie tapissait le sol. Des panneaux carrés de 45 centimètres de còté sont juxtaposés. chacun d'eux formés de piêces assemblées avec soin avec aul centre une rosace. Il est a noter que certaines pierres sont taillées a angle droit. mais que d'autress suivent des courbes complexes.

A l'Ouest, on peut distinguer plusieurs groupes de salles disposés autour de cours it (iel ouvert (LXIII, LXXXIV et vaiscmblablement LXXIII). A l'angle sudtouest, bien des pieces ont disparu. du fait de l'elfondement des voùtes qui less supportaient. Le serul espace bien conservé est l'espace l.XIII. Cne double rangée de murs entoure ce rect angrte assez irrégulier. Ies murs "xtérieurs doivent etre ceux de la cour ; les murs intérieurs porlaient sans doute une colonnade. Au centre de cet espace, un petil massif carré en briques et tuiles d'où sort un tuyau de plemb vertical : sans doute un jet d'reau. La canalisation qui vient de l'angle nord-est est arrachée. mais sa trace est visible. Tout aulour le sol était pavé de carrés el de rectangles de marbere of de schistes noirs qui alternaient. Il reste 
peu de plaques en place. mais de dessin est net. La difficulté est de restituer l'aspect de la cour en bordure du péristyle car le carrelage ne se retrouve plus : vers le Nord. le mur de la rolonnade supposéc est bordé par un béton de tuileau; vers l'Ouest. celui-ci porte des plaques dargile cuite qui ont pu porter une mosaïque ?). Entre ces éléments et le parage. un vide tout autour de la pièce : 'cmme si l'on avait arraché une décoration. une bordure? Quant au couloir qui fait le tour de cet espace, il devait ètre couvert de béton où se mèlent des fragments de marbre semés irrégulièrement. Il en subsiste une certaine surface au Nord.

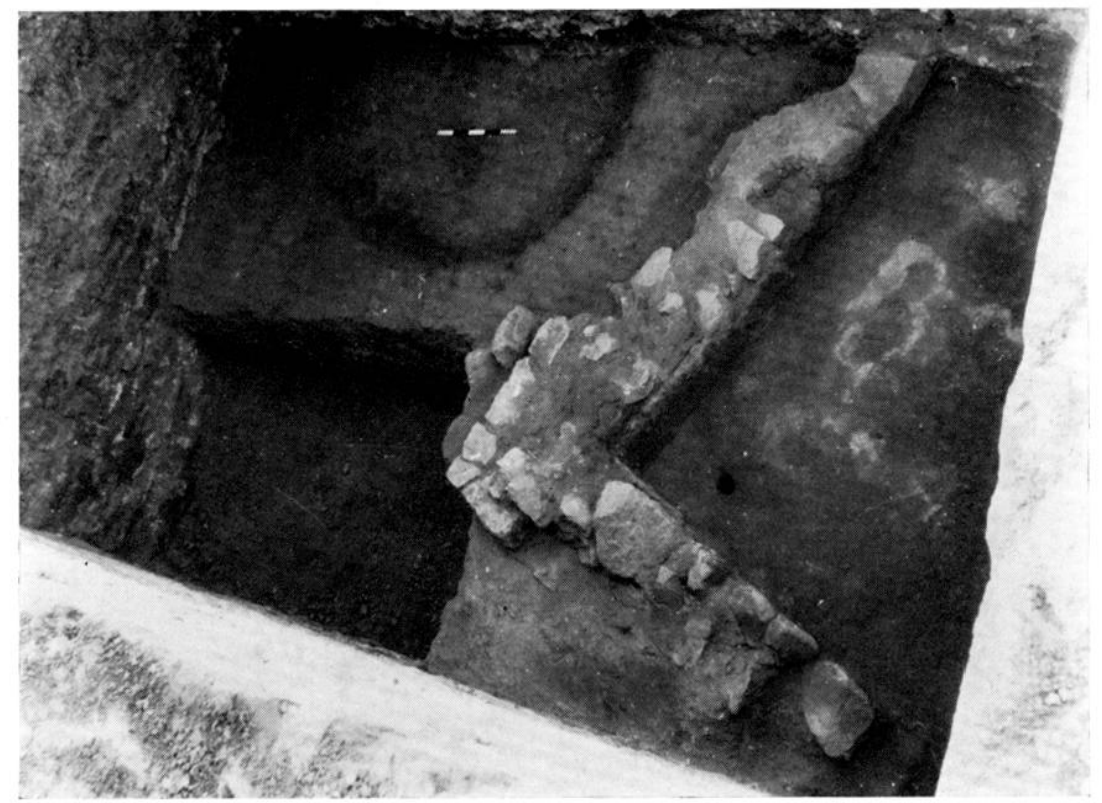

l:ig. 21. -. - l.e four de polier. A ganche, en haut, construction circulaire voir fier. :2:)!.

En remontant vers le Nord. une pièce LXIX allongée en hordure de la cour centrale. Elle conserve une partie de sa mosaïque a petits cubes noirs. Des pièces placées plus a l'Ourst. seuls les couloirs se distinguent (IXY et LXVI). La salle LXIV s'ot partiellement écroulée. Elle est traversée par un égout. Il reste dans son mur nord un passage de $1 \mathrm{~m}$. 70 qui mène vers IXXIV. le sol de béton de cette ouverture est bien conservic at de part et d'autre on voit encore la trace de deux niches semi-circulaires logées dans le mèm" mur.

La restitution des salles retrouvées sur la face ouest dans l'axe de la cour centrale est très conjecturale. On trouve d'abord en venant de l'Est. trois pièces (LXX. LXXI. IXXII) puis un grand espace LXXIII flanqué de deux constructions allongées (IXXIV at I.XXX). Enfin au fond, dans un premier état, de petites niches rectangulaires IIXXY. LXXVI. LXXVII. LXXVIII. LXXIX) accolées au mur latéral occidental de la plate-forme. Toutes ces constructions n'existent qu'en fondation et elles ont entièrement perdu leur sol. Le seul niveau est donné par le seuil déjà signalé entre LXIV et LXXIV. Vess le milieu de LXXIII est placée une petite pièce voùtée qui parail bien une citerne. S'il en est ainsi, il est rraisemblable de voir dans ce grand espace une rour.

Quant à l'abside qui est ménagée dans l'axe du monument. vers l'ouest. elle e'st posté- 


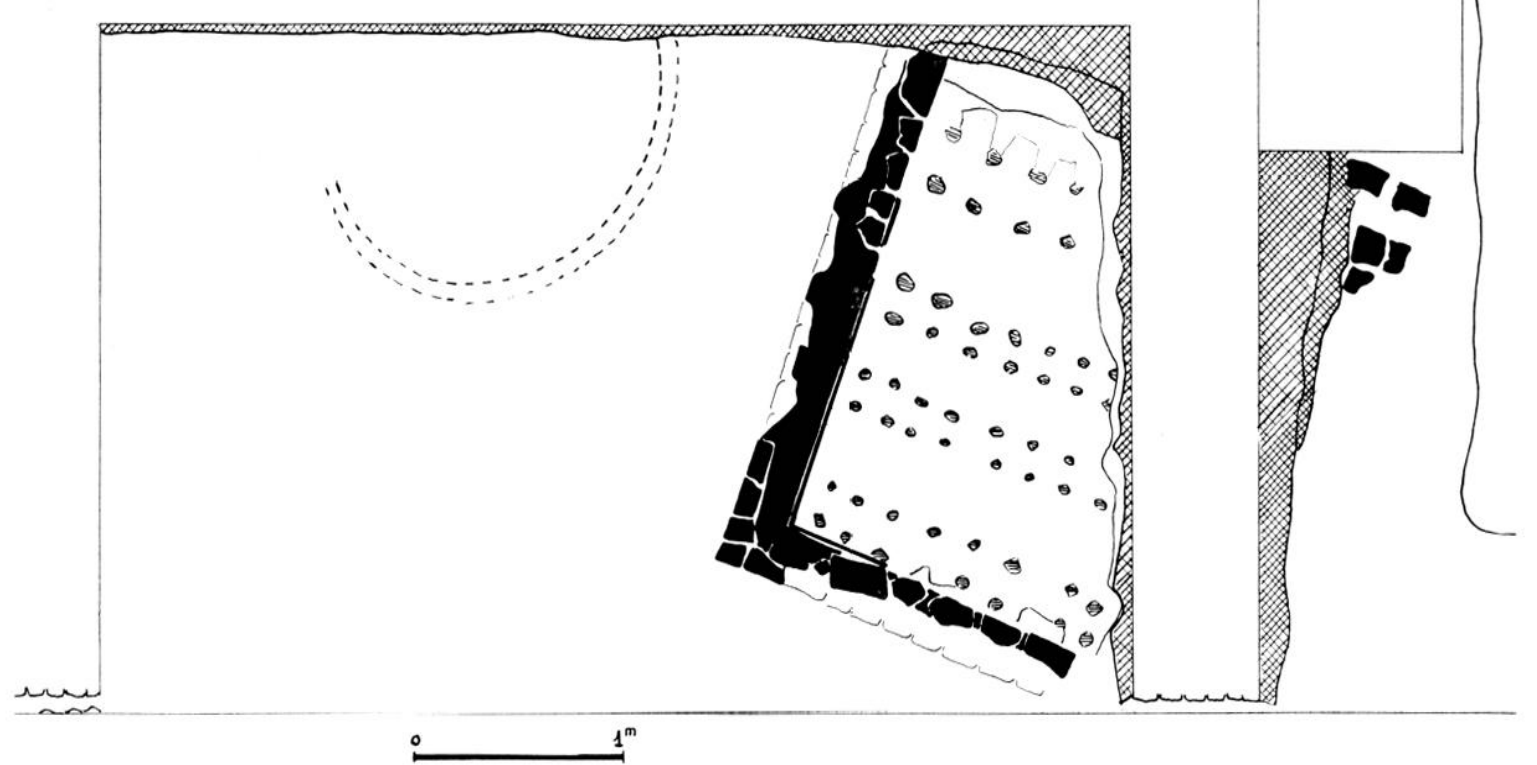

Pig. :2:. - Plan sommaire des parties degagies du four. A ganuche, four (?) circulaire (voir lig. : I ).

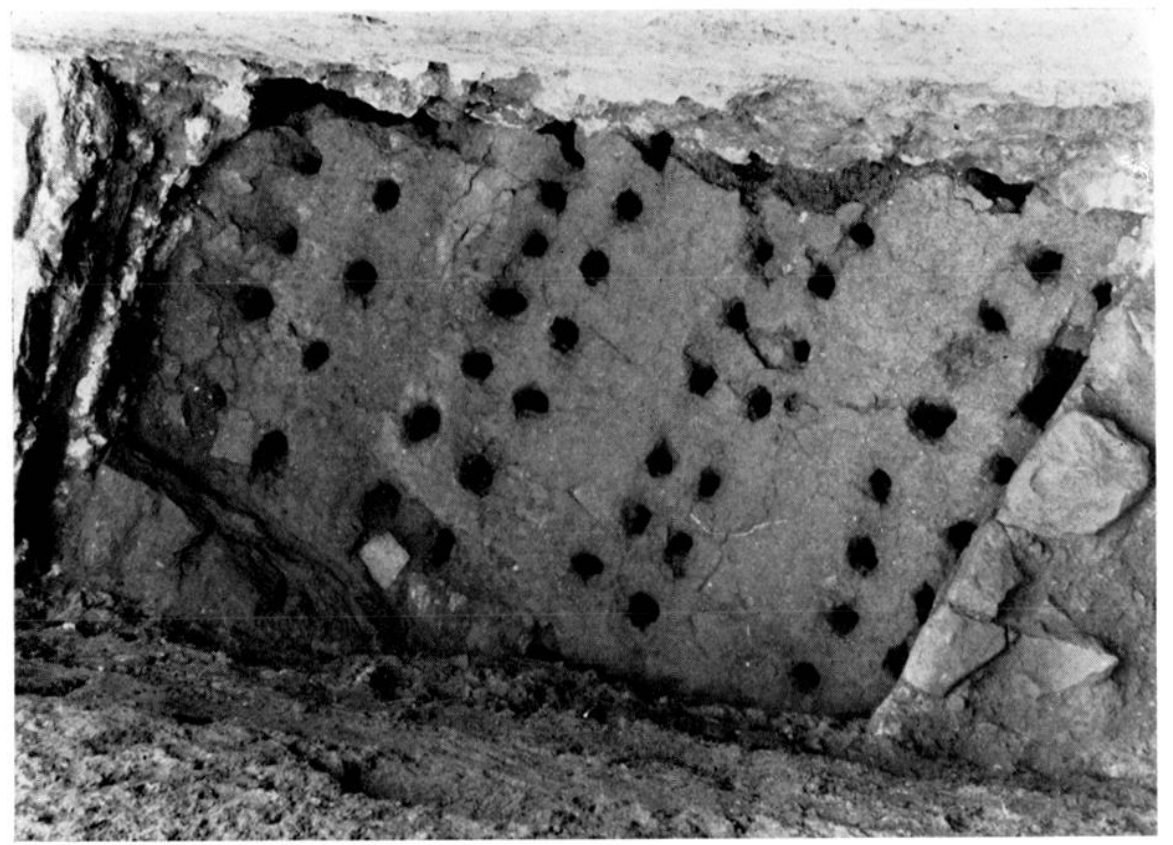

Firg. 2:3. - l.a sole du four.

rieure à cel ensemble. En refret, ses fondations ont été creusés dans la terere de remblai : de la leur irrégularité. Les autres murs présentent par contre un appareil. en fondation. irrégulier, mais aux joints lissés à la truelle : ils ont été bàtis arant le comblement ef le nivellement. Comme cette zone avait été fouillée par le Dr Donnarlieu. je n'ai pu relever d'autres indiens chronologique's.

A l'angle nord-ouest de la Plate-Forme, des pieress sont aussi disposés autour d'un 


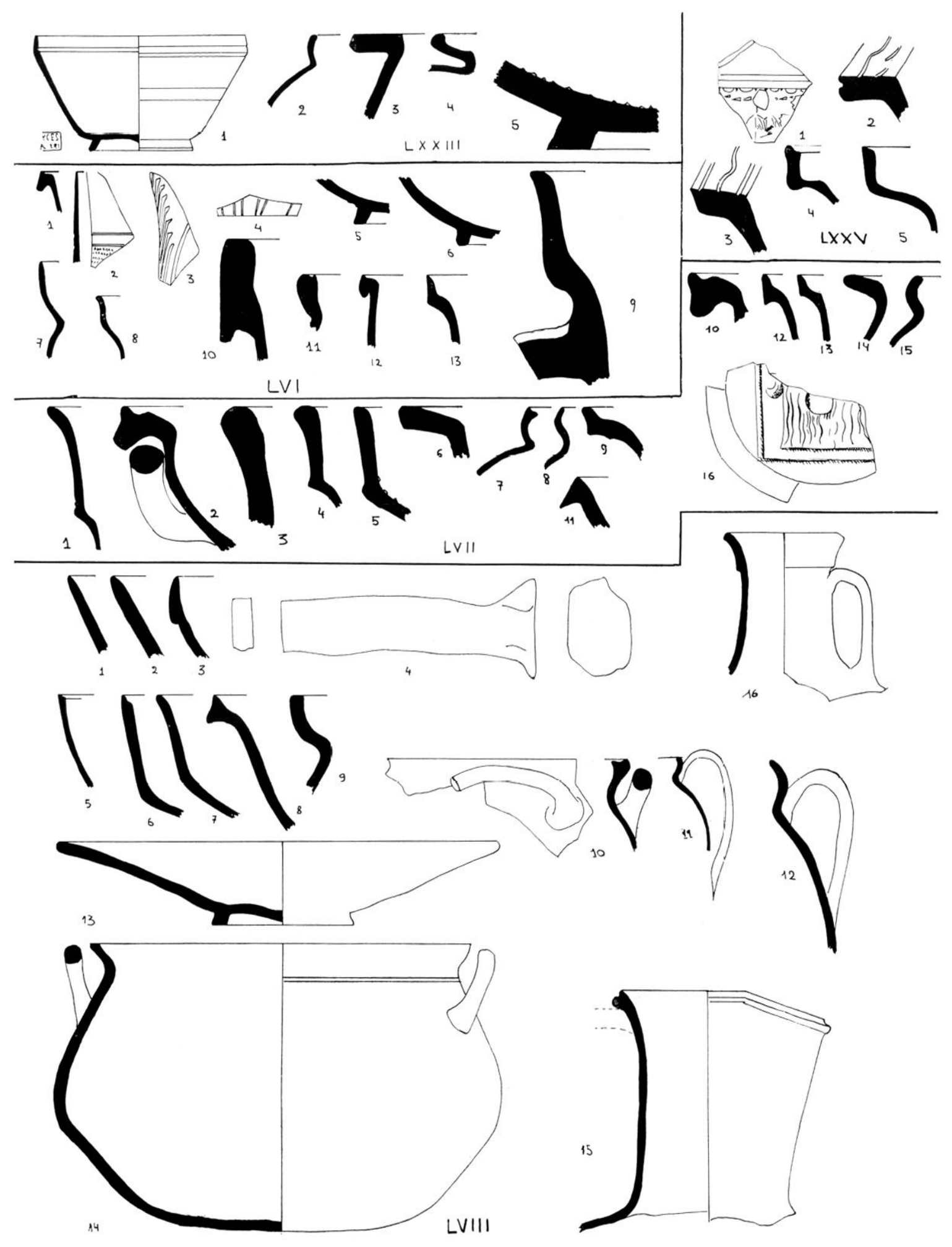

Fig. 24. - Caramique antérieure à la construction de la Plate-Forme. 
espace sensiblement ('arré (13 m. 2) sur $13 \mathrm{~m}$. 84 aver un couloir sur les quatre fares: sans doute est-ce-là encore une cour à péristyle. De l'angle sud-ouest de cet espace ouvert. part un égout qui se dirige vers le Sud-Ouest et sort du terre-plein dans l'axe de la cour rentrale. En ce point, il rencontre un autre égont venu du sud-Et, mais dont l'origine n'a pas encore éte reconnue. Parmi ces pièces, seules di et cill ont encore des restes d'un sol an béton.

\section{Sondage dans le lerre-plein.}

Sous les constructions a l'Est de la cour centrale. less sondages ont donné partout une terre stérile : sans doute est-ce dì au fait que les battiments ont été édifiés sans qu'il füt nécessaire de remblayer. Par contre. vers l'(Ouest, lo remblai est très important. Il était logique de penser que ers terres pouraient contenir quelques tessons el, de fait, bien que la récolte ait été assez maigre. eu égard au volume de terre déplacé. d'intéressants indices ont été trouvés. Ie plus, un dess sondages a montré que le terrain était occupé avant la construction de la Plate-Forme : un four de potier a élé reconnu. Voici les principaux éléments recueillis ${ }^{23}$. qui proviennent tous des couches de remblais amenés immédiatement après la construction du réseau des fondations et antérieurs aux sols de l'édifice primitif de la Plate-Forme. (ies remblais sont des sables ou des aruiles vertes ou rouges (fig. :24).

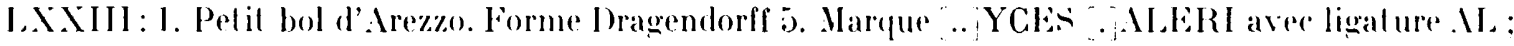
ellacée a cauche; sans doule 'cr yces l"aleri (4730):

2. Rebord de pol (?) polerie noire tres cuile micalce (4tio) ;

3. Rebord polerie noire miracée (1733) :

4. Rebord poterie noire écrasée à la cuisson (4736i);

¿). Fond de morlier : poterie grise ( 17331$)$.

LXXV : 1. Fragment de vase décoré d'Arezzo (4152);

2. Rebord de grand plat a marli. polerie jaune lendre (4273);

3. Rebord de grand plat a marli, poterie orangée mieux cuile (4289);

4. Rebord de large pot (?) a pàle ocre (4292);

i. Rebord de cruche à pate orangée (4298).

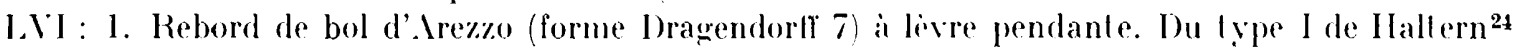
$(4097)$;

2. Rebord de gobelet i parois fines, paite rougeatre vernis rouge, incisions (4081);

3. Fragment de gobelet à parois fines. pàte orangée. incisions (4101);

4. Fond de gobelet a parois fines. paite orangée, incisions (3702);

5. Fond de bol (?) d'imilation de campanienne. paite ocre clair micarée, vernis noir conserves ¿l l'exlérieur (10135) :

6. Fond de grand bol, imil at ion de campaniemne, paile orangée micacée, vernis noir ou rougesil re par endroils $(3676,36,16,4079)$;

7. Rebord de cruche. pate orangée micacéc, couverle rougeatere imilant l'arétine;

8. Rebord de cruche, campanienne $\mathrm{C}$ (-11089);

9. Fragment de col d'amphore, pàte jaunâtre ì grains de dégraissant, micacée (3677. 1157 :

10. Rebord de col d'amphore. pàle jaunaltre plus fine (3642);

(23) Dans ce rapport préliminaire, on ne trouvera qu'un choix. Une publication d'ensemble de ce mat riel suiva après achevement des fonilles. Il seral e'n effet indispensable de publier le matériel d'une conche anssi bien homogine

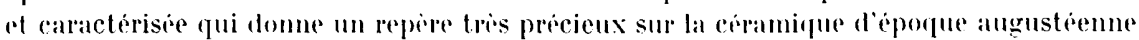

(2.4) A rapprocher d'un bol trouré sur la Butte Saint-Antoine: Gallia, t. XIT, 1956, p. 49, tig. 15, 1" L.X, 3. 
11. Rebord de pol, poterie grise rugueuse (36i39):

12. Rebord de pol. poterie orangée (34)8; ;

13. Rebord de cruche (?) polerie orangée (369):

L.VIl : 1. Col de cruche à pàle orangée: bec l réllé (42.2):

2. Fragment de grand plat d'environ f0 centimelres de diametre supérieur. anse incurvée. pale grise $(3745)$;

3. Rebord de col d'amphore, pàte ocre (3783. 377:) ;

4. Rebord pâte ocre micacée $(3170)$ :

Ђ. Rebord de mortier, pàte ocre micarée (380.4);

6. Rehord de grand vase pate orangée (3801);

7. Rebord de grand pot. pâte orangee wranuleuse micacée $(3780)$;

8. Rebord de pot. pate ore (3814):

9. Rebord de wrand vase, pale ocre $(3765)$;

10. Rebord de grand rase. paite ocre plus fine $(3731)$;

11. Rebord de grand vase. pate ocre lendre $(3742)$;

12 i 15 . Rebords de pols, pàle grise granuleuse (3776. 4132. 3802. 4119);

16. Fragment de grand rase (?) ou d'oscillum. paile orangée micacée, représentant un masque (38:27 a :38:31:

Thans la salle IVI. le sondage avait montré que les fondations avaient détruit un mur antérieur dont il ne restait que quelques pierres. Le sondage en LVII a fait retrouver d'autres murs, ceux d'un four qui n'a pas été complètement fouillé au terme de la campagne' de 1961. Les murs sont en petit appareil irrégulier lié par une argile rouge. Les parois sont enduites d'argile qui a cuit. La sole rectangulaire détruite vers l'Est est percée de rangées de trous irréguliers. Actuellement, l'alendier n'a pas été dégagé (fig. :21-2:2).

Le niveau de la sole est à $3 \mathrm{~m}$. 30 environ du pavement de la salle de la plate-forme. Le comblement a été obtenu d'abord avec les matériaux provenant de la destruction du four. puis en amenant des remblais de l'extérieur. De la couche la plus basse qui doit correspondre' a la dernière époque du four. de nombreux tessons ont été sortis:

LVII : 1. Imilation de campanienne. pàte ocre, vernis brillant rouge brun inlerne, brun noir exlerne (i) 406 (6):

2. Imilalion de campanienne. pàte orangée. vernis rouge brun el noiràtre interne ef externe (5)(1)48);

3. Rebord de large coupe. pàte orangée avec surface légèrement plus rouge ;

4. Outil de potier (?) en argile ocre (5372);

5. Rebord de bol en poterie noire. fine. bien lissee (5373. 5376);

6. Rebord de grande jalte pàte granuleuse rougreâtre à grains de dégraissant micacée (4832), 48.35 , etc.);

7. Rebord de grande jatte paite semblable (4971);

8 . Rebord de grande jatte. pàte ocre micacée (4848);

9. Rebord pàte ocre granuleuse à wrains de dégraissant (4867);

10. Fragment de marmite à anse aplatie (5) $57,5114,4750)$. paile noirail re granuleuse ;

11. Rebord de pol. pàte semblable (475) ;

12. Rebord de pol. pàte semblable (53361);

13. Plats, pàte rougeàtre granuleuse (4708. 4787);

14. Marmite. pâte semblable (5620), 5622):

15. Col de cruche it bec tréflé. pàte semblable (oxil9?: 
16. Col d'amphore, paile jaunàtre (4694, 496;3 it 4968) ${ }^{25}$.

Noter en outre un fragment de masque semblable a I.VII, 16.

Il semble que les vases de céramique commune 6 a 8,10 a 15 . quils soient de paite rougeàtre ou noiràtre, sortent d'un mème atelier. seul le degré de cuisson a dì différencier les objets. En effet. plusieurs cols à bec tréflé ont été sortis. en pàte noiràtre. semblables au no 15. Il n'est pas possible encore de déterminer quelle a été la production du four : loriques, tuiles ou rases (et lesquels?).

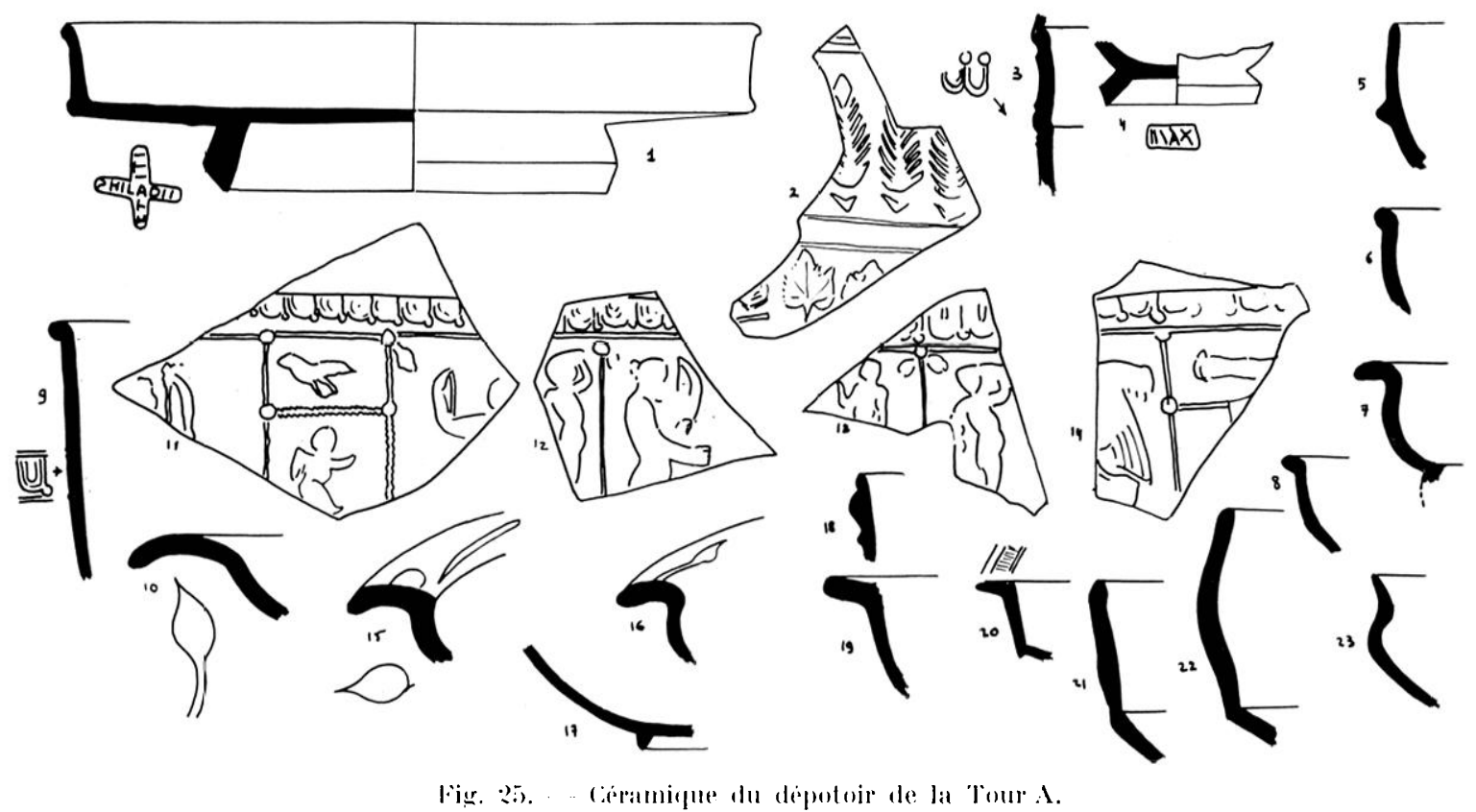

\section{An Vord de la Plate-Forme.}

Plusieurs salles ont été partiellement dégagées (elles sont sur le chemin d'évacuation des terres) au Nord du mur de façade du battiment de la Plate-Forme contre lequel elles se sont appuyées. l'est de là que partent deux égouts qui vont se jeter sans doute dans l'égout qui a été signalé parallèle à la façade, plus vers l'ouest. Aucun matériel n'a encore été trouvé qui permette de dater ces pièces, qui sont pourtant postérieures a la façade.

La tour de l'enceinte urbaine a été trouvée comblée par un important dépotoir. Maluré la présence de trois courhes (deux couches grisatres séparées par une courhe noire plus ou moins épaisse at très pulvérulente), il semble diflicile de distinguer une réelle chronologie. Les objets les plus lourds (cols d'amphores) ont tous éte décourerts au-dessus de l'argile verte qui marque le niveau primitif.

Les principaux élements de datation de eette rouche sont regroupes sur les figures 2) (at $: 6$.

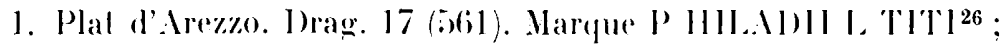

(25); Gallia, 1. . XI1, 1956, p. 51, lig. $16,: 2$.

:(6) Rapprocher : (.I.I., t. 1;, 6700) 688 et 711-712. 
FOCILLES IIE FRÉJU:
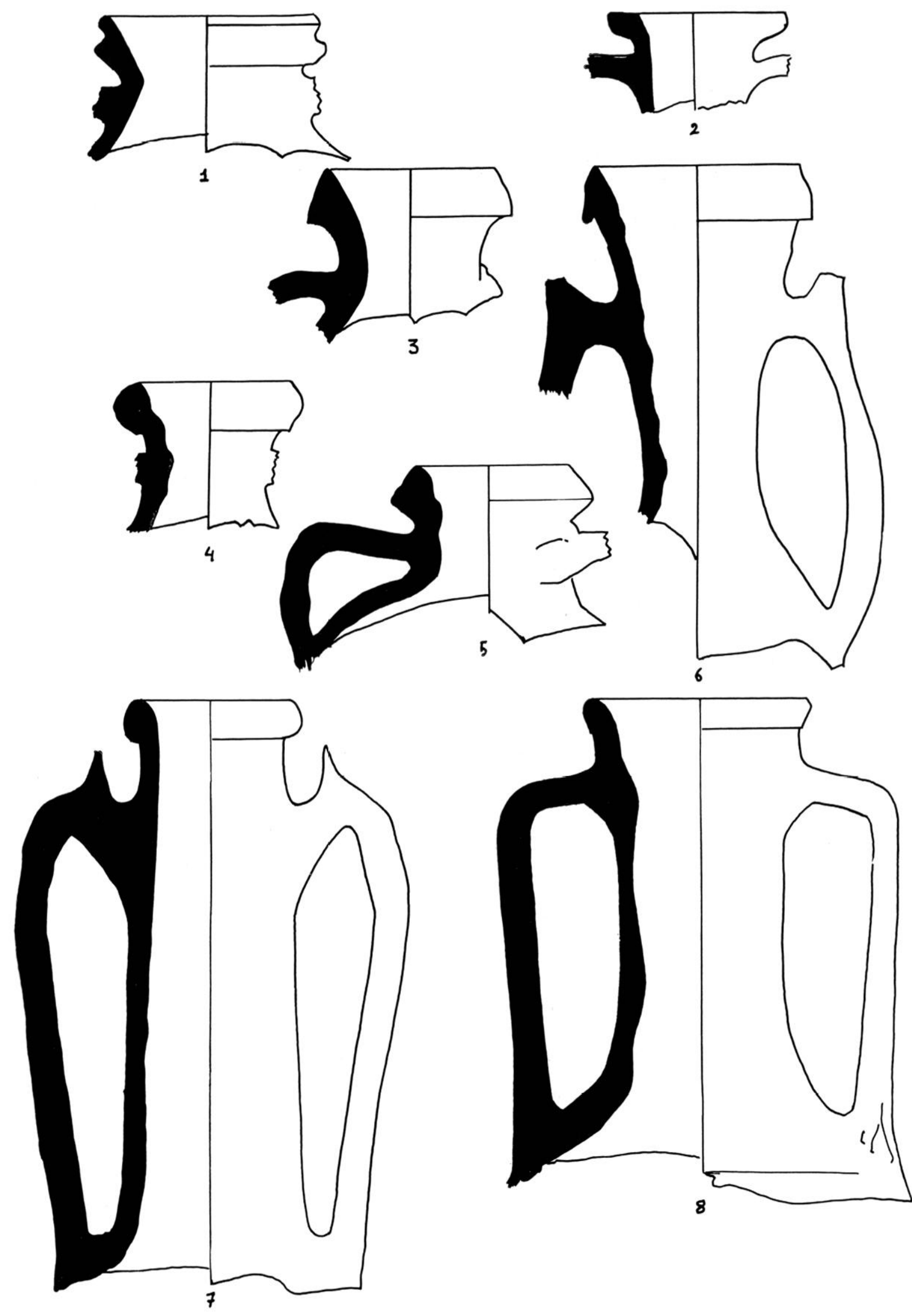

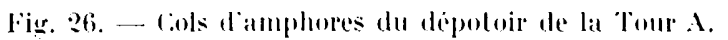


2. Fragment de vase caréné d'Arezzo proche de la forme Gose 7 (1184);

3. Fragment d'Arezzo (1345);

4. Fond de coupelle d'Arezzo. Marque II.XX (944);

5. Rebord de coupelle du sud de la Gaule (386) l)rag. 25);

6. Rebord de coupelle du sud de la Gaule (715);

7. Rebord de coupelle du sud de la Gaule avec décor de feuilles d'eau (1176). Drag. 3.);

8. Fragment de rebord du Sud de la Gaule (952);

9. 11 a 14. Fragments d'un grand vase du sud de la Gaule. Mrag. 37. A impressions tres sommaires ; décor de métopes. Victoire. I)iane chasseresse. Amours. Oiseaux (186. 197, 546);

10. Rebord de grand plat du sud de la (iaule. Drag. 36 (5):5) ;

15. Rebord de coupe de sigillée claire A. Forme Lamboglia 4/36 (494);

16. Autre fragment voisin de mème pàte (804);

17. Fond de coupe de sigillée claire $\mathrm{A}$. forme $4 / 36(721)$;

18. Fragment de rebord de sigillée claire .1 . trop cuite el qui a viré au brun (979);

19. Rebord de sigillée claire $A$. Peut-ètre forme $6(562)$;

20. Rebord de sigillée claire B. Forme inédite (949);

21 el 22. Rebords de plats en céramique " préluisante " (872.871);

23. Coupelle vitrifiée; pâte jaunâtre à l'intérieur, verdâtre à l'extérieur (608).

La présence de la céramique préluisante et de sigillée claire B, ainsi que l'élément de roupelle à parois vitrifiées, font penser que le dépotoir est postérieur à la fin du il $\mathrm{e}^{\mathrm{e}}$ siècle. Or le ressaut de fondation d'un mur placé entre cette tour, l'eneeinte et la façade de la Plate-Forme, coüncidait aver la limite supérieure de ce dépotoir. Ies murs dont il a été question au paragraphe précédent sont-ils postérieurs au ${ }_{11} \mathrm{e}$ siècle?

\section{¿). Conclusions.}

De ce rapport préliminaire sur deux rampagnes de fouilles de 1960 et 1961, un certain nombre de conclusions se dégagent qui amènent à poser divers problèmes.

Ine partie de l'enceinte de la ville avee deux de ses tours se trouve dégagée. Mais l'état de la ruine à l'angle sud-est du terrain empêche d'expliquer la raison et le plan romplet du saillant que ce mur faisait à l'origine. Contre cette enceinte, et après un certain temps, a été bàti un grand bâtiment à cour centrale dont la construction a entraîné de grands remblais pour établir sur la butte primitive un terre-plein artificiel.

Sur cette butte, un habitat de potiers existait avant l'édification de la Plate-Forme. La présence de potiers à Fréjus ne peut surprendre étant donné la présence de riches giserments d'argile mème sur les banes de grès qui portent la ville romaine (argile blanche scmblahle à celle du dépòt encore exploité a Bellevue, immédiatement à l'list de la cité). Ce qui est important e'est que nous retrouvons là une nouvelle trace du Forum .Julii antérieur à l'époque augustéenne ou du tout début de cette époque. Déja les fouilles de la Butle Saint-Antoine on 195i) avaient fait retrouver dans un sondage une construction détruite lors de l'édification de ce monument ${ }^{27}$. On mesure done par là l'extension de l'agrglomération de part et d'autre du port. Quelle est la date exacte de ce premier habital ? Il est encore impossible d'ètre précis. le qui parait rependant vraisemblable. à en juger par le matériel à ce jour dégagé (vases d'Arezzo du service I le Haltern. gohelets à parois

$27 \quad$ Gallia, t. NIT, 1956, p. $47-19$. 
fines. campanienne (i, imitation de campanienne, vaisselle commune parfois comparable). r'est que les deux monuments de la Butte Saint-Antoine et de la Plate-Forme sont sensiblement contemporains. Le premier est. on le sait, postéricur à 44-4? avant notre ère. Aucune monnaie n'a encore été trouvée en stratigraphie dans le second. Contentons-nous de dire que les deux monuments sont d'époque augustéenne et qu'ils ont été édifiés sur un site déjà habité.

Lorsque le four de potier fonctionnait, était-il dans l'enceinte ? Celle-ci était-clle déjà construite ? Il sera difficile de répondre à cette question car la date de l'enceinte est pour le moment inconnue et les sondages faits à ses abords n'ont apporté aucun indice.

In autre problème sans solution est celui de la fonction du monument de la PlateForme. Le I) ${ }^{\mathrm{r}}$ Donnadieu arait pensé y retrouver le prétoire. Nais il est à noter que cette lıypothèse est antérieure à toute fouille, car il l'a exprimée dès $1927^{28}$ et cet archéologue s'est efforcé de rérifier cette idée dans la suite de ses publications. Il est nécessaire de dire que la plupart des identifications faites par ce chercheur à qui Fréjus doit tant, sont douteuses et certaines mèmes impossibles (ainsi l'auguralorium).

Les deux plans de la Butte Saint-Antoine et de la Plate-Forme sont très proches: une cour centrale rectangulaire; autour, des salles qui se groupent autour d'autres cours plus petites; un large espace vide du còté du port (jardin ?). Nous connaissons trop mal la vie de la cité à l'époque augustéenne et l'épigraphie ne nous renseigne pas sur la vie de la (ité et ses institutions. L'examen du plan de la Plate-Forme nous force à rejeter le terme de ritadelle, car ie monument n'a de valeur défensive que vers l'Est, là où est l'enceinte de la cité ; ailleurs il n'en a aucune, son mur de façade au Nord étant de 40 centimètres de large et les cares à l'Ouest largement ouvertes. Les contreforts du mur sud le montrent bien. De ce monument on disposait d'une large vue sur la rade et le port. La vaste surface occupée, la complexité du plan suggèrent qu'il y a là un bâtiment public. Je ne crois pas pouvoir en dire davantage.

Ciet édifice a été remanié, au moins partiellement, jusqu'au $\mathrm{II}^{\mathrm{e}}$ siècle. J'ai relevé seulement un tesson de céramique estampillée rouge et une monnaie du rve siècle. Il faut se garder d'utiliser l'argument a silentio car la surface du terrain a été tellement endommagée par les labours et les plantations (des oliviers au siècle dernier) que toute trace de remaniement a pu disparaitre.

Les prochaines campagnes de fouilles ne nous aideront certainement pas à mieux connaître la fin du monument de la Plate-Forme; mais elles nous apporteront sans aucun doute de précieuses indications sur les origines de Fréjus.

Paul-Albert FÉvrier.

(28) La Pompei de la Prolence, Fréjus, Paris, 1927, p. 32. 